\title{
Limited Information and Advertising in the US Personal Computer Industry
}

\author{
Michelle Sovinsky Goeree ${ }^{1}$
}

First version: June, 2002

This version: January, 2008

\begin{abstract}
Traditional discrete choice models assume buyers are aware of all products for sale. In markets where products change rapidly the full information assumption is untenable. I present a discrete choice model of limited consumer information, where advertising influences the set of products from which consumers choose to purchase. I apply the model to the US personal computer market where top firms spend over $\$ 2$ billion annually on advertising. I find estimated markups of $19 \%$ over production costs, where top firms advertise more than average and earn higher than average markups. High markups are explained to a large extent by informational asymmetries across consumers, where full information models predict markups of one-fourth the magnitude. I find that estimated product demand curves are biased towards being too elastic under traditional models. I show how to use data on media exposure to improve estimated price elasticities in the absence of micro ad data.
\end{abstract}

JEL Classification: L15, D12, D21, M37, L63

Keywords: Advertising, information, discrete choice models, product differentiation, personal computer industry

\footnotetext{
${ }^{1}$ University of Southern California and Claremont McKenna College (email: michelle.goeree@gmail.com). This paper is based on my 2002 dissertation. Special thanks to my advisors, Steven Stern and Simon Anderson. I am grateful to Costas Meghir and three anonymous referees for their detailed comments, which substantially improved the paper. The paper has benefited from comments of seminar partipants at Amsterdam, Arizona, Claremont McKenna, Edinburgh, KU Leuven, Southern California, Tilburg, UC Irvine, Virginia, Warwick, Yale, EARIE meetings, and IIOC meetings and discussions with Dan Ackerberg, Steve Berry, Greg Crawford, Jacob Goeree, Phil Haile, Mike Keane, Aviv Nevo, Margaret Slade, Matt Shum, Frank Verboven, and Michael Waterson. I thank Gartner Inc. and Sandra Lahtinen for making the data available. I am grateful for financial support from the University of Virginia's Bankard Fund for Political Economy.
} 


\section{Introduction}

In 1998 over 36 million personal computers (PCs) were sold in the US, generating over $\$ 62$ billion in revenues- over $\$ 2$ billion of which was spent on advertising. The PC industry is one in which products change rapidly, with approximately 200 new products introduced by the top 15 firms every year (Gartner Inc., 1999). Due to the large number of PCs available and the frequency with which new products are brought into the market, consumers are unlikely to be aware of all PCs for sale. Furthermore, it is reasonable to suspect consumers have limited information in many industries.

Traditional random coefficient discrete choice models are estimated under the assumption that buyers are aware of all available products. Within the full information framework, Berry, Levinsohn, and Pakes (1995)(hereafter BLP) show that it is important to allow for consumer taste heterogeneity in order to obtain realistic estimates of demand elasticities. This paper adds to BLP and shows that it is just as important to allow for heterogeneity in consumer information in industries with a rapidly changing product line. Indeed, in rapidly changing markets informational asymmetries may explain (perhaps a significant) part of the variation in sales.

This paper presents a model of limited information where the imperfect substitutability between different brands may arise from limited consumer information about product offerings as well as from idiosyncratic brand preferences. The limited information model incorporates three important sources of consumer heterogeneity: choice sets, tastes, and advertising media exposure. Following the data combining approach of Petrin (2002), I show how to estimate a model of limited information in the absence of micro-level advertising data, which are difficult to obtain in many industries. ${ }^{1}$

The results suggest that traditional models, which rule out non-random informational asymmetries a priori, can yield estimates of product-specific demand curves that are biased towards being too elastic. The estimates indicate that advertising has very different informative effects across individuals and media, and that allowing for heterogeneity in consumer information yields more realistic estimates of demand elasticities.

The results show that (i) limited information about a product is a contributing factor to differences in purchase outcomes and (ii) information is distributed across households in a non-random way. An implication of these findings is that assuming full information may lead to incorrect conclusions regarding the intensity of competition. Indeed, I found high estimated median markups in the PC industry in 1998, about 19\%, whereas traditional full

\footnotetext{
${ }^{1}$ Recent structural studies of advertising utilizing micro purchase and advertising exposure data include Erdem and Keane (1996), Ackerberg (2003), and Anand and Shachar (2004). Shum (2004) matches aggregate advertising data to micro purchase data.
} 
information models suggest the industry was more competitive, with estimated markups of only 5\%. Furthermore, the results suggest top firms benefit from limited consumer information with the top firms earning higher than average markups and engaging in higher than average advertising. These implications are of particular importance when addressing policy issues.

The paper proceeds as follows, in the next section I describe the data. I discuss the model and identification in sections 3 and 4 . Estimation is discussed in section 5 . The results from preliminary regressions and from the full model are presented in sections 6 and 7, respectively. I describe the specification tests and conclude in the final sections 8 and 9 .

\section{Data}

Product Level Data The product level data were provided by Gartner Inc. and consist of quarterly shipments and dollar sales of all PCs sold between 1996 and 1998. ${ }^{2}$ The majority of firms sell to the home market, businesses, educational institutions, and the government. Since the focus of this research is on consumer behavior, I use the home market data to estimate the model. ${ }^{3}$ Sales to the home market comprise over $30 \%$ of all PCs sold.

As can be seen from Table I, the PC industry is concentrated, with the top six firms accounting for over $69 \%$ (71\%) of the dollar (unit) home market share on average. The major market players did not change over the period, although there was significant change in some of their market shares. The top ten firms, based on home market share (Acer, Apple, Compaq, Dell, Gateway, Hewlett-Packard, IBM, Micron, NEC, and Packard-Bell), account for over $80 \%$ of PC sales to the home market. The analysis includes the top ten firms and five others (AST, AT\&T / NCR, DEC, Epson, and Texas Instruments) to make full use of micro-purchase data. ${ }^{4}$ The 15 "included" firms account for over 85\% (83\%) of the dollar (unit) home market share on average.

I have data on five main PC attributes: manufacturer (e.g. Dell), brand (e.g. Latitude LX), form factor (e.g. desktop), CPU type (e.g. Pentium II), and CPU speed (MHz). I define a model as a manufacturer, brand, CPU type, CPU speed, form factor combination. Due to data limitations, I do not include some essential product characteristics (such as memory or hard disk) or product peripherals (such as CD-ROM or modem). However, the

\footnotetext{
${ }^{2}$ Prices are dollar sales divided by units sold and are deflated using the Consumer Price Index from BLS.

${ }^{3}$ I use the non-home sector data in the supply side of the model (see section 3.3).

${ }^{4}$ While all firms were active in 1996, by 1998, Texas Instruments had merged with Acer, DEC had merged with Compaq, and the other three firms had disappeared from the home market. I treat changes in number of products and firms as exogenous variation, a common assumption made in this literature. I discuss the impact of including the smaller firms on the results in section 8 .
} 
ease with which consumers can add on after purchase (by buying RAM or a CD-ROM, for instance) would make it difficult to determine consumer preferences over these dimensions. The data I use consist of a more limited set of attributes, but those which cannot be easily altered after purchase. The Gartner data still allow for a very narrow model definition. For example, the Compaq Armada 6500 and the Armada 7400 are two separate models. Both have Pentium II 300/366 processors, 64 MB standard memory, 56KB/s modem, an expansion bay for peripherals, and full-size displays and keyboards. The 7400 is lighter, although somewhat thicker, and it has a larger standard hard drive, and more cache memory. In both models the hard drive and memory are expandable up to the same limit. In addition, the Apple Power Macintosh Power PC 604 180/200 desktop and deskside are two separate models. They differ only in their form factor.

\begin{tabular}{|c|c|c|c|c|c|c|c|c|}
\hline \multirow{3}{*}{ Manufacturer } & \multicolumn{3}{|c|}{ Percentage Dollar } & \multicolumn{3}{|c|}{ Average Annual } & \multicolumn{2}{|c|}{ Median Percentage Markup } \\
\hline & Hon & Market & & $\mathrm{Ad}$ & Ad to Sales & Median Price & over Marginal Costs & including ad costs \\
\hline & 1996 & 1997 & 1998 & Expend & Ratio & Home Sector & Home Sector & Home Sector \\
\hline Industry & & & & & $3.4 \%$ & $\$ 2,239$ & $15 \%$ & $10 \%$ \\
\hline Top 6 Firm & 65.67 & 68.31 & 75.26 & $\$ 469$ & $9.1 \%$ & $\$ 2,172$ & $17 \%$ & $12 \%$ \\
\hline Acer & 6.20 & 6.02 & 4.37 & $\$ 117$ & $5.4 \%$ & $\$ 1,708$ & $11 \%$ & $9 \%$ \\
\hline Apple & 6.66 & 5.79 & 9.16 & $\$ 161$ & $5.3 \%$ & $\$ 1,859$ & $16 \%$ & $9 \%$ \\
\hline AST & 3.08 & 1.53 & & & & & $13 \%$ & \\
\hline Compaq & 11.89 & 16.29 & 16.43 & $\$ 208$ & $2.4 \%$ & $\$ 2,070$ & $23 \%$ & $16 \%$ \\
\hline Dell & 2.46 & 2.87 & 2.57 & $\$ 150$ & $2.1 \%$ & $\$ 2,297$ & $10 \%$ & \\
\hline Gateway & 8.94 & 11.77 & 16.43 & $\$ 277$ & $5.6 \%$ & $\$ 2,767$ & $12 \%$ & $10 \%$ \\
\hline Hewlett-Packard & 4.02 & 5.52 & 10.05 & $\$ 651$ & $17.7 \%$ & $\$ 2,203$ & $16 \%$ & $10 \%$ \\
\hline IBM & 8.49 & 7.42 & 6.85 & $\$ 1,189$ & $20.1 \%$ & $\$ 2,565$ & $16 \%$ & $10 \%$ \\
\hline Micron & 3.26 & 4.05 & 1.68 & & & & $7 \%$ & \\
\hline NEC & 3.22 & & & & & & & \\
\hline Packard Bell & 23.48 & & & & & & & \\
\hline Packard Bell - NEC & & 21.02 & 16.33 & $\$ 327$ & $7.2 \%$ & $\$ 2,075$ & $16 \%$ & $11 \%$ \\
\hline Texas Instruments & 1.40 & & & & & & $7 \%$ & \\
\hline 15 included & 83.11 & 82.27 & 83.88 & & & & & \\
\hline
\end{tabular}

Notes:Others in the 15 included are ATT(NCR), DEC, and Epson, each of which held less than 1\% of the home (and total) market shares in 1996 and 1997 . AST and Micron held less than $1 \%$ total market shares on average. In 1997 three mergers occurred :Packard Bell, NEC,ZDS; Acer,Texas Instr.; Gateway, Advanced Logic Research. Ad expenditures (in $\$ M$ ) and ad to sales ratios are annual averages and are from LNA and include all sectors (home, business, education, government). Percentage markups are the median (price-marginal costs)/price across all products. The last column is percentage total markups per unit after including advertising. These are determined from estimated markups and estimated effective product advertising in the home sector.

Table I: Summary Statistics for Market Shares, Advertising, Prices, and Markups

Treating a model/quarter as an observation, the sample size is $2112,{ }^{5}$ representing 723 distinct models. The majority of the PCs offered to home consumers were desk PCs (70\%) and over $83 \%$ of the processors were Pentium-based. The number of models offered by each firm varied. Compaq had the largest selection with 138 different choices, while Texas Instruments offered only five. On average, each firm offered a model for three quarters.

The market size is the number of US households in a given period, as reported by the Census Bureau. Market shares are unit sales of each model divided by market size. The outside good market share is one minus the share of the inside goods.

\footnotetext{
${ }^{5}$ This is the sample size after eliminating observations with negligible quarterly market shares.
} 
Advertising Data Due to data limitations, previous studies were unable to consider the differential effects of advertising across media. I use advertising data from Competitive Media Reporting's (CMR) LNA/ Multi-Media publication, which includes quarterly ad expenditures across ten media. Some of the media channels are not used frequently by PC firms. For example, outdoor advertising for PCs is rare (on average less than $0.3 \%$ of ad expenditures). I aggregate the media into newspaper, magazine, television (TV) and radio categories. ${ }^{6}$ These broader channels contain more non-zero observations aiding identification of media specific parameters.

These data are not broken down by sector (e.g. home, business, etc.). CMR categorizes advertising across product types, which, in some instances, allows me to isolate non-home expenditures. For example, some expenditures are reported with detail, (e.g. IBM RS/6000 server) while others are generally reported (e.g. IBM various computers). As a result, the ad measure includes some expenditures on non-PC systems intended for non-home sectors (such as mainframe servers and UNIX workstations).

Total ad expenditures by the top firms in the computer industry have grown from $\$ 1.4$ billion in 1995 to over $\$ 2$ billion in 1998 (an average annual rate close to 13\%). As Table I shows, there is much variation across firms. The industry ad-to-sales ratio is $3.4 \%$. However, the top firms spend on average over $9 \%$ of their sales revenue on advertising. Notably, the majority of the top firm expenditures are by IBM whose ad-to-sales ratio is over $20 \%$. IBM's large relative ad expenditures may be due to its non-PC interests (servers, mainframes, UNIX workstations, etc.). To examine this hypothesis, in the model, I allow the position of the firm in the non-PC sector to affect the non-home sector marginal revenue of advertising. Excluding IBM's expenditures, the remaining top firms spend an average of $6.5 \%$ of their revenue on advertising. In contrast, Compaq's ad-to-sales ratio is only $2.4 \%$.

It is common for PC firms to advertise products simultaneously in groups. For example, in 1996, one of Compaq's ad campaigns involved all Presarios (of which there are 12). One possibility is that group advertising provides as much information about the products in the group as product-specific advertising. However, if group advertising were as effective as product advertising, we would observe only group advertising (the most efficient use of resources). An alternative possibility is that group advertising merely informs the consumer about the firm. If this were the case, we should observe either firm-level (the largest possible group) or product-specific advertising.

In reality, firms use a combination of product-specific and group advertising (with groups of varying sizes). I need a measure of ad expenditures by product that incorporates all

\footnotetext{
${ }^{6}$ The "magazine" medium includes Sunday magazines. The "television" medium includes network, spot, cable or syndicated TV. The "radio" medium includes network and spot radio. There are many zero observations for outdoor advertising, and so I choose to add it to the radio medium.
} 
advertising done for the product. I construct "effective" product ad expenditures by adding observed product-specific expenditures to a weighted average of all group expenditures for that product where the weights are estimated. Let $\mathcal{G}_{j}$ be the set of all product groups that include product $j$ (I suppress the time subscript). Let $a d_{\mathcal{H}}$ be (observed) total ad expenditures for group $\mathcal{H} \in \mathcal{G}_{j}$ where the average expenditure per product in the group is

$$
\overline{a d}_{\mathcal{H}} \equiv \frac{a d_{\mathcal{H}}}{|\mathcal{H}|}
$$

Then "effective" ad expenditures for product $j$ are given by

$$
a d_{j}=\sum_{\mathcal{H} \in \mathcal{G}_{j}}\left(\pi_{1} \overline{a d}_{\mathcal{H}}+\pi_{2} \overline{a d}_{\mathcal{H}}^{2}\right)
$$

where the sum is over the different groups that include product $j .{ }^{7}$ This specification allows for increasing or decreasing returns to group advertising. If there is only one product in the group (i.e. it is product-specific), I restrict $\pi_{1}$ to unity and $\pi_{2}$ to zero.

Consumer Level Data The consumer level data come from the Survey of Media and Markets conducted by Simmons Market Research Bureau. Simmons collects data on consumers' media habits, product usage, and demographics from about 20,000 households annually. Ideally, one would have individual-level purchase, ad exposure, and demographic data. Unfortunately, these data are not available for the PC industry. However, I am able to use the Simmons data to link demographics with purchases and to control for household variation in advertising media exposure. I use two years of the survey from 1996-1997 (data from 1998 were not publicly available). Descriptive statistics are given in Table II. ${ }^{8}$

The Simmons respondents were asked about their media habits. I use the self-reported media exposure information to control for variation in advertising media exposure across households. I combine the Simmons data with (separate) information on market shares and product characteristics, which enables me to obtain a more precise picture of how media exposure and demand are related. I use these data to construct "media exposure" moments.

In addition, Simmons collects information on PC ownership, including whether the individual purchased in the past year and the manufacturer. Approximately $11 \%$ of the households purchased a PC in the last 12 months. Respondents were not asked any specifics

\footnotetext{
${ }^{7}$ I call these "effective" product ad expenditures to indicate they are constructed from observed group and product-specific advertising. To get an idea of the level of detail in the data: in the first quarter of 1998, there were 18 group advertisements for Apple computers. The groups advertised ranged from "various computers" to "PowerBook" to "Macintosh Power PC G3 Portable" (the later being a specific model). In this quarter the Apple Macintosh Power PC G3 Portable computer belonged to 7 different product groups.

${ }^{8}$ The Simmons survey oversamples in large metropolitan areas. This causes no estimation bias because residential location is treated as exogenous. To reduce the sample to a manageable size, I select 6700 respondents randomly from each year. The final sample size is 13,400.
} 
regarding their $\mathrm{PC}$ other than the manufacturer. Only the 15 firms used in estimation were listed separately. I use these data to construct "firm choice" moments.

\begin{tabular}{|c|c|c|c|c|}
\hline \multirow[t]{2}{*}{ Variable Description } & \multicolumn{2}{|c|}{ Sample } & \multicolumn{2}{|c|}{ Population } \\
\hline & Mean & Std. Dev. & Mean & Std. Dev. \\
\hline male & 0.663 & 0.474 & 0.661 & 0.473 \\
\hline white & 0.881 & 0.324 & 0.881 & 0.324 \\
\hline age (years) & 47.38 & 15.68 & 46.87 & 15.13 \\
\hline 30 to50 ( $=1$ if $30<$ age $<50)$ & 0.443 & 0.497 & 0.449 & 0.497 \\
\hline education (years) & 13.98 & 2.54 & 14.00 & 2.35 \\
\hline married & 0.564 & 0.496 & 0.572 & 0.495 \\
\hline household size & 2.633 & 1.429 & 2.631 & 1.428 \\
\hline employed & 0.695 & 0.460 & 0.693 & 0.461 \\
\hline income $(\$)$ & 56745 & 45246 & 56340 & 44465 \\
\hline inclow $(=1$ if income $<\$ 60,000)$ & 0.667 & 0.471 & 0.669 & 0.471 \\
\hline inchigh ( $=1$ if income $>\$ 100,000)$ & 0.107 & 0.309 & 0.106 & 0.308 \\
\hline own pc (=1 if own a PC) & 0.466 & 0.499 & 0.470 & 0.499 \\
\hline pcnew (=1 if PC bought in last 12 months) & 0.113 & 0.317 & 0.112 & 0.316 \\
\hline media exposure & Mean & Std. Dev. & Min & Max \\
\hline cable ( $=1$ if receive cable) & 0.749 & 0.434 & 0 & 1 \\
\hline hours cable (per week) & 3.607 & 2.201 & 0 & 7 \\
\hline hours non-cable (per week) & 3.003 & 2.105 & 0 & 6.2 \\
\hline hours radio (per day) & 2.554 & 2.244 & 0 & 6.5 \\
\hline magazine ( $=1$ if read last quarter) & 0.954 & 0.170 & 0 & 1 \\
\hline number magazines (read last quarter) & 6.870 & 6.141 & 0 & 95 \\
\hline weekend newspaper (=1 if read last quarter) & 0.819 & 0.318 & 0 & 1 \\
\hline weekday newspaper (=1 if read last quarter) & 0.574 & 0.346 & 0 & 1 \\
\hline
\end{tabular}

Table II: Descriptive Statistics for Simmons Data

Finally, I use data on the distribution of consumer characteristics from the Consumer Population Survey (CPS) in the macro moments. Unlike Simmons, the CPS data are available from 1996-1998. ${ }^{9}$ I discuss the media exposure micro moments, the firm choice micro moments and the macro moments in section 5.1.

\section{Economic Model}

The model primitives are product attributes, consumer preferences, and the notion of equilibrium. In the product and ad-level data, I observe price, quantity, other measurable product attributes, and ad expenditures across media. In the consumer-level data, I observe consumer attributes, including media exposure, and firm choice. The structural estimation strategy requires me to specify a model of consumer choice and firm behavior and derive the implied relationships among choice probabilities. ${ }^{10}$

\footnotetext{
${ }^{9}$ For each year I drew 3,000 individuals from the March CPS. Quarterly income was constructed from annual data and deflated using the Consumer Price Index. I dropped a few households where annual income was below $\$ 5000$. Simmons data indicate that no purchases were made by households with income below $\$ 5000$, hence eliminating these households should not affect the group of interest.

${ }^{10}$ The model is static, primarily due to lack of micro data on purchases and ad exposure. A static model does not capture long-term advertising effects, such as brand building. While brand building is important,
} 


\subsection{Utility and Demand}

An individual chooses from $J$ products, indexed $j=1, \ldots, J$, where a product is a PC model defined as a firm-brand-CPU type-CPU speed-form factor combination. Product $j$ characteristics are price $(p)$, non-price observed attributes $(x)$ (CPU speed, Pentium CPU, firm, laptop form factor, etc.), and attributes unobserved to the researcher but known to consumers and producers $(\xi) .{ }^{11}$ The indirect utility consumer $i$ obtains from $j$ at time $t$ is

$$
u_{i j t}=\delta_{j t}+\mu_{i j t}+\epsilon_{i j t}
$$

where $\delta_{j t}=x_{j}^{\prime} \beta+\xi_{j t}$ captures the base utility every consumer derives from $j$ and mean preferences for $x_{j}$ are captured by $\beta .{ }^{12}$ The composite random shock, $\mu_{i j t}+\epsilon_{i j t},{ }^{13}$ captures heterogeneity in consumers' tastes for product attributes, and $\epsilon_{i j t}$ is a mean zero stochastic term distributed i.i.d. type I extreme value across products and consumers.

The $\mu_{i j t}$ term includes interactions between observed consumer attributes $\left(D_{i t}\right)$, unobserved (to the econometrician) consumer tastes $\left(\nu_{i}\right)$, and $x_{j}$. Specifically,

$$
\mu_{i j t}=\alpha \ln \left(y_{i t}-p_{j t}\right)+x_{j}^{\prime}\left(\Omega D_{i t}+\Sigma \nu_{i}\right) \quad \nu_{i} \sim N\left(0, I_{k}\right) .
$$

The $\Omega$ matrix measures how tastes vary with $x_{j}$. I assume that $\nu_{i}$ are independently normally distributed with a variance to be estimated. $\Sigma$ is a scaling matrix. Income is $y_{i t}$.

Consumers have an "outside" option, which includes nonpurchase, purchase of a used $\mathrm{PC}$, or purchase of a new $\mathrm{PC}$ from a firm not in the 15 included firms. Normalizing $p_{0 t}$ to zero, the indirect utility from the outside option is

$$
u_{i 0 t}=\alpha \ln \left(y_{i t}\right)+\xi_{0 t}+\epsilon_{i 0 t}
$$

I also normalize $\xi_{0 t}$ to zero, because I cannot identify relative utility levels.

\subsection{Information Technology}

In industries where new product introductions are frequent, the full information assumption is not innocuous. This paper considers a model of random choice sets, where the probability

the majority of PC firms have not changed over the period and most had been in existence for many years prior to 1996. These firms would not have as much need to establish a brand image as to spread information about new products. The static framework permits me to focus on the influence of advertising on the choice set absent the additional structure and complications of a dynamic setting. Also, the nature of advertising in the PC industry lends itself to a static framework. Products change rapidly, and the effects of advertising today on future information provision are minimal since the same products are no longer for sale.

${ }^{11}$ I do not include brand fixed effects because there are over 200 brands.

${ }^{12}$ Note that this indirect utility can be derived from a Cobb-Douglas utility function (see BLP).

${ }^{13}$ Choices are invariant to multiplication by a person-specific constant, so I fix the standard deviation of $\epsilon_{i j t}$. Since there are over 2000 products estimating an unrestricted covariance matrix is not feasible. 
that consumer $i$ purchases product $j$ depends upon the probability she is aware of $j$, the probability she is aware of the other products competing with $j$, and the probability she would buy $j$ given her choice set. ${ }^{14}$ Assuming consumers are aware of the outside option with probability one, the (conditional) probability that consumer $i$ purchases $j$ is

$$
s_{i j t}=\sum_{\mathcal{S} \in \mathcal{C}_{j}} \prod_{l \in \mathcal{S}} \phi_{i l t} \prod_{k \notin \mathcal{S}}\left(1-\phi_{i k t}\right) \frac{\exp \left\{\delta_{j t}+\mu_{i j t}\right\}}{y_{i t}^{\alpha}+\sum_{r \in \mathcal{S}} \exp \left\{\delta_{r t}+\mu_{i r t}\right\}}
$$

where $\mathcal{C}_{j}$ is the set of all choice sets that include product $j$. The $\phi_{i j t}$ term is the probability $i$ is informed about $j$. The $y_{i t}{ }^{\alpha}$ term is from the presence of the outside good. The outside sum is over all the choice sets that include product $j$.

One could consider calculating (3) directly for each individual, which would require computing all purchase probabilities corresponding to each possible choice set. If there were three products, one could easily calculate the four purchase probabilities associated with each choice set for each individual. Given the large number of products in the PC industry $(J=2112)$, it is not feasible to calculate the $2^{J-1}$ purchase probabilities corresponding to each choice set for each individual and product. Obviously, if one observed the choice set then the computational burden would be substantially eased. Unfortunately, these data are not available. A solution to the computational problem is to simulate the choice set facing $i$, thereby making only one purchase probability computation per individual necessary: the one corresponding to $i$ 's simulated choice set. I implement this solution and provide details in section 5.2. Therefore, the choice set facing an individual is a simulated one and hence is not observed directly from the data, rather the data used to form the choice sets are those used to construct the $\phi_{i j t}$ term, which I now discuss.

The information technology, $\phi_{i j t}$, describes the effectiveness of advertising at informing consumers about products. Suppressing time notation, it is given by

$$
\phi_{i j}\left(\theta_{\phi}\right)=\frac{\exp \left(\gamma_{j}+\lambda_{i j}\right)}{1+\exp \left(\gamma_{j}+\lambda_{i j}\right)}
$$

which is a function of medium advertising where the $m=1, \ldots, M$ media are magazines, newspapers, television, and radio. The $m$ th element of the $M \times 1$ vector $a_{j}$ is the number of ads for $j$ in $m .{ }^{15}$ The components of $\phi_{i j}$ that are the same for all consumers is given by

$$
\gamma_{j}=a_{j}^{\prime}\left(\varphi+\rho a_{j}+i_{m} \Psi_{f}\right)+\vartheta x_{j}^{a g e}
$$

\footnotetext{
${ }^{14}$ Leslie (2004) presents a discrete-choice model with random choice sets. In his model consumers choose seat quality at a Broadway play. Patrons receive a coupon, which gives them the opportunity to purchase a high quality ticket at a discount, with a certain probability.

${ }^{15}$ The number of advertisements in medium $m$ are advertising expenditures, $a d_{j m}$, divided by the weighted average price of an advertisement in medium $m$. Recall from equation (1) that $a d_{j m}$ is a weighted sum of model specific and group advertising where the weights, $\pi_{1}, \pi_{2}$, are to be estimated.
} 
where the vectors, $\varphi$ and $\rho$, measure the effectiveness of advertising media at informing consumers. I include fixed effects for those firms that offered a product every quarter (the $\Psi_{f}$ ), but do not estimate a fixed effect for each medium, so $i_{m}$ is a column vector of ones. Finally, consumers may be more likely to know a product the longer it has been on the market, this is captured by $\vartheta$ where $x_{j}^{a g e}$ is the PC age measured in quarters.

Ideally, one would have individual ad exposure data. Unfortunately, these data are not available for many industries including the PC industry. I control for variation in household ad exposure (as it is related to observables) by using media exposure information from Simmons. The $\lambda_{i j}$ captures consumer information heterogeneity:

$$
\lambda_{i j}=a_{j}^{\prime}\left(\Upsilon D_{i}^{s} \zeta+\kappa_{i}\right)+\widetilde{D}_{i}^{\prime} \widetilde{\lambda} \quad \ln \kappa_{i} \sim N\left(0, I_{m}\right)
$$

The $\Upsilon$ matrix captures how advertising media's effectiveness varies by observed consumer characteristics. Simmons data are used to identify $\Upsilon$, where $D^{s}$ is a larger set of demographic characteristics from the Simmons data. ${ }^{16}$ Thus $\Upsilon_{m} D_{i}^{s}$ is the exposure of individual $i$ to medium $m$, and $a_{j}^{\prime} \Upsilon D_{i}^{s}$ is the exposure of $i$ to ads for product $j$. The parameter $\varsigma$ measures the effect of this ad exposure on the information set. The $\kappa_{i}$ vector are unobserved (to the econometrician) consumer heterogeneity with regard to ad medium effectiveness. ${ }^{17}$ I assume $\kappa$ are independent of other unobservables.

In the absence of advertising, consumers still may be (differentially) informed (i.e. $\phi(a=$ $0)>0$ ). The $\widetilde{D}$ (a subset of $D$ ) proxy for the opportunity costs of acquiring information. ${ }^{18}$ The magnitude of $\phi_{i j}$ when no advertising occurs depends on $\widetilde{D}_{i}^{\prime} \widetilde{\lambda}+\vartheta x_{j}^{a g e}$.

Notice $\phi_{i j}$ depends upon own product advertising only. Allowing informational spillovers would greatly complicate the model. First, the theoretical framework would have to address free-riding in advertising choices across firms. Second, one would need adequate variation in the data to empirically identify the spillover effect across products. For these reasons, I assume the probability a consumer is informed about a product is (conditional on her attributes) independent of the probability she is informed about any other product. Information provided (via advertising) for one product (or by one firm) cannot "spillover" to another product (or to another firm). That is, I assume product or group advertising for product $r \neq j$ provides no information about $j$.

Let $\varkappa_{i}=\left(y_{i}, D_{i}, \nu_{i}, \kappa_{i}\right)$ be the vector of individual characteristics. I assume that the consumer purchases at most one good per period, ${ }^{19}$ that which provides the highest utility, $U$, from all the goods in her choice set. Let $R_{j} \equiv\left\{\varkappa: U\left(\varkappa, p_{j}, x_{j}, a_{j}, \xi_{j}, \epsilon_{i j}\right) \geq\right.$

\footnotetext{
${ }^{16}$ There are 11 demographic characteristics included in $D^{s}$. These are measures of age, household size, marital status, income, sex, race, and education.

${ }^{17}$ To limit the number of parameters to estimate, I normalized the variance of the $\kappa$ to one for all media.

${ }^{18}$ These consist of dummies for high school graduate, income $<\$ 60,000$, and income $>\$ 100,000$.

${ }^{19}$ This assumption may be unwarranted for some products for which multiple purchase is common. How-
} 
$\left.U\left(\varkappa, p_{r}, x_{r}, a_{r}, \xi_{r}, \epsilon_{i r}\right) \quad \forall r \neq j\right\}$ define the set of variables that results in the purchase of $j$ given the parameters of the model. The home market share of product $j$ is

$$
s_{j}=\int_{R_{j}} d G(y, D, \nu, \kappa, \epsilon)=\int_{R_{j}} s_{i j} d G_{y, D}(y, D) d G_{\nu}(\nu) d G_{\kappa}(\kappa)
$$

where $G(\cdot)$ denotes the respective distribution functions. The second equality follows from independence assumptions. The conditional probability that $i$ purchases $j, s_{i j}$, is given in (3). Market share is a function of prices and advertising of all products. The smaller is $\phi_{i j}$, the smaller is product market share. If $\phi_{i j}$ were equal to one for all products, market share would be the standard full information choice probability. ${ }^{20}$ Demand for $j$ at time $t$ is $\mathcal{M}_{t} s_{j t}$, where $\mathcal{M}_{t}$ is the market size given by the number of households in the US.

\subsection{Firm Behavior}

I include the supply side for a few reasons. First, firms often advertise products in groups. The model of demand requires a measure of product advertising that incorporates all advertising done for the product. I construct effective product ad expenditures that is a weighted average of group ads for that product with estimated weights $\left(\pi_{1}\right.$ and $\left.\pi_{2}\right)$. Supply side moments are used to identify the weights. Second, following BLP, I use information from the first order conditions to estimate marginal costs, which allows me to calculate markups. Finally, I compare my model to benchmark cases. The supply side helps to more precisely estimate some of the parameters in the benchmark models.

I assume there are $f=1, \ldots, F$ non-cooperative, Bertrand-Nash competitors. Each firm produces a subset of the $J$ products, $\mathcal{J}_{f}$. Suppressing time notation, profits of firm $f$ are

$$
\sum_{j \in \mathcal{J}_{f}}\left(p_{j}-m c_{j}\right) \mathcal{M} s_{j}(p, a)+\sum_{j \in \mathcal{J}_{f}} \Pi_{j}^{n h}\left(p^{n h}\right)-\sum_{m} m c_{j m}^{\mathrm{ad}}\left(\sum_{j \in \mathcal{J}_{f}} a_{j m}\right)-\mathcal{C}_{f}
$$

where $s_{j}$ is home market share given in $(5) ; m c_{j}$ is marginal cost of production; $\Pi_{j}^{n h}$ is gross profit (before advertising) from the non-home sectors; $p^{n h}$ is price in the non-home sector; $m c_{j m}^{\text {ad }}$ is marginal cost of advertising in medium $m$; and $\mathcal{C}_{f}$ are fixed costs of production.

Following BLP, I assume $m c_{j}$ are log-linear and composed of unobserved $\left(\omega_{j}\right)$ and observed $\left(w_{j}\right)$ cost characteristics and parameters to be estimated $(\eta)$. I expect $\omega_{j}$ to be

ever it is not unreasonable to restrict a consumer to purchase one computer per quarter. Hendel (1999) examines purchases of PCs by businesses and presents a multiple-choice model of PC purchases.

${ }^{20}$ Grossman and Shapiro (1984)(GS) present a theoretical circle model in which ad messages provide information about product availability. The empirical model presented here differs along several dimensions: (i)I allow for a more flexible model of differentiation and estimate a discrete choice model (Anderson, et al.,1989); (ii)unlike GS, consumers may be informed if there is no advertising; (iii)I do not observe individualspecific ad messages, which is central to GS; (iv)once a consumer is aware of the product she is also aware of its attributes. Hence, the information technology (and market shares) differ from GS. 
correlated with $\xi_{j}$ because PCs with high unobserved quality might be more expensive to produce. I account for the correlation in estimation. The (log) marginal cost function is

$$
\ln \left(m c_{j}\right)=w_{j}^{\prime} \eta+\omega_{j}
$$

I assume $m c_{j m}^{\text {ad }}$ are composed of observed components, $w_{j m}^{a d}$ (such as the average price of an ad), ${ }^{21}$ and unobserved components, $\tau_{j}$. The (log) marginal cost of advertising in $m$ is

$$
\ln \left(m c_{j m}^{\mathrm{ad}}\right)=w_{j m}^{a d \prime} \psi+\tau_{j} \quad \tau_{j} \sim N\left(0, I_{m}\right)
$$

where $\psi$ is to be estimated. I set the variance of $\tau_{j}$ to one for all media channels. ${ }^{22}$

Given their products and the advertising, prices, and attributes of competing products, firms choose prices and advertising media levels simultaneously to maximize profits. Product attributes that affect demand $\left(x_{j}, \xi_{j}\right)$ and those that affect marginal costs $\left(w_{j}, \omega_{j}, w_{j m}^{a d}, \tau_{j}\right)$ are treated as exogenous to price and advertising decisions. ${ }^{23}$ Firms may sell to home and non-home sectors. Constant marginal costs imply pricing decisions are independent across sectors. $^{24}$ Any product sold in the home sector will have prices that satisfy

$$
s_{j}(p, a)+\sum_{r \in \mathcal{J}_{f}}\left(p_{r}-m c_{r}\right) \frac{\partial s_{r}(p, a)}{\partial p_{j}}=0 .
$$

However, an advertisement intended to reach a home consumer may affect sales in other sectors. Optimal advertising choices must equate the marginal revenue of an additional advertisement in all sectors with the marginal cost. Advertising medium choices satisfy

$$
\mathcal{M} \sum_{r \in \mathcal{J}_{f}}\left(p_{r}-m c_{r}\right) \frac{\partial s_{r}(p, a)}{\partial a_{j m}}+m r_{j}^{n h}=m c_{j m}^{\mathrm{ad}}
$$

where $m r^{n h}$ is the marginal revenue of advertising in non-home market sectors. Specifically, $m r_{j}^{n h}=\theta_{p}^{n h} p_{j}^{n h}+x_{j}^{n h \prime} \theta_{x}^{n h} \cdot{ }^{25}$ Characteristics of product $j$ sold in the non-home sector are

\footnotetext{
${ }^{21}$ The CMR data consist of ad expenditures across ten media. The quarterly average ad price in media group $m$ is a weighted average of ad prices in the original categories comprising the group $m$. The weights are firm specific and are determined by the distribution of the firms advertising across the original media.

${ }^{22}$ Computational constraints dictate I choose which are the more interesting parameters to estimate.

${ }^{23}$ Adequately addressing the issue of endogenous product characteristics would require a dynamic model of the process that generates product characteristics. This topic is beyond the scope of this paper.

${ }^{24}$ Pricing decisions may not be independent across sectors (if the price of a particular laptop is lower for business, a consumer might buy the laptop from their business account for use at home). Identification of a model which includes pricing decisions across all sectors would require richer data for non-home sectors. Also, education, business, and government groups usually purchase multiple PCs, which greatly complicates the model (Hendel, 1999). While the assumptions that I impose imply independent pricing decisions, the estimates are sensible, and goodness-of-fit tests suggest the model fits the data reasonably well.

${ }^{25}$ Ideally, one would construct $m r^{n h}$ in a structural framework. Identification would require much richer data and one should allow for multiple purchases. The $m r^{n h}$ could also depend on rivals' prices and advertising, this would increase the estimation burden and require more of the advertising data. Since my focus is on the home sector, I approximate the $m r^{n h}$ with the simplified specification above.
} 
price $\left(p_{j}^{n h}\right)$ and other observable characteristics $\left(x_{j}^{n h}\right)$ including advertising, CPU speed, and non-PC firm sales. ${ }^{26}$ The $\theta^{n h}$ are parameters to be estimated. Let $\eta_{\mathrm{AD}}=\left\{\operatorname{vec}(\psi), \operatorname{vec}\left(\theta^{n h}\right)\right\}$.

\section{Identification}

Following the literature, I assume that the demand and pricing unobservables (evaluated at the true parameter values, $\left.\Theta_{0}\right)$ are mean independent of a set of exogenous instruments, $z$ :

$$
E\left[\xi_{j}\left(\Theta_{0}\right) \mid z\right]=E\left[\omega_{j}\left(\Theta_{0}\right) \mid z\right]=0 .
$$

I do not observe $\xi_{j}$ or $\omega_{j}$, but market participants do. This leads to endogeneity problems because prices and ad choices are most likely functions of unobserved characteristics. If price is positively correlated with unobserved quality, price coefficients (in absolute value) will be understated (as preliminary estimates in section (6) indicate). Whereas if advertising is positively correlated with quality, its effect will be overstated. ${ }^{27}$

A solution involves instrumental variables. ${ }^{28}$ BLP show that variables that shift markups are valid instruments for price in differentiated products models. In a limited information framework the components of $z$ include the characteristics of all the products marketed (the $x$ ), variables that determine production costs (the components of the $w$ that are not in $x$ ) and variables that determine advertising costs (the components of $w^{a d}$ ). ${ }^{29}$ The value of the instrument for any given product can be any function of $z$.

The intuition to motive the advertising instruments is similar to that used by BLP to motivate the price instruments. Products which face more competition (due to many rivals offering similar products) will tend to have lower markups relative to more differentiated products. Advertising for $j$ depends on $j$ 's markup. As ad first order conditions (FOC) in (10) indicate, a firm will advertise a product more the more they make on the sale of the product, ceteris paribus. The pricing FOCs in (9) show the optimal price (and hence markup) for $j$ depends upon characteristics of all of the products offered. Therefore, the optimal price and advertising depends upon the characteristics, prices, and advertising of all products offered. Note also that the level of advertising for $j$ in media $m$ depends on

\footnotetext{
${ }^{26}$ Non-PC sales are constructed by subtracting quarterly PC sales from quarterly total manufacturer sales (as recorded in firm quarterly reports). Therefore "non-home sales" include sales of computer systems such as mainframes, servers, and UNIX workstations.

${ }^{27}$ See Milgrom and Roberts (1986).

${ }^{28}$ Berry (1994) was the first to discuss the implementation of instrumental variables methods to correct for endogeneity between unobserved characteristics and prices. BLP provide an estimation technique. My model and estimation strategy is in this spirit but is adapted to correct for advertising endogeneity.

${ }^{29}$ Variables that determine production costs that are not in $x$ include a time trend. Hence, production costs shifters do not play a large role in identifying demand in the model presented in section (3).
} 
the marginal cost of advertising in that media. Thus the instruments will be functions of attributes, product cost shifters, and advertising cost shifters of all other products.

Given (11) and regularity conditions, the optimal instrument for any disturbance-parameter pair is the expected value of the derivative of the disturbance with respect to the parameter (evaluated at $\Theta_{0}$ ) (Chamberlain, 1987). Optimal instruments are functions of advertising and prices. To use the optimal instruments, I would have to calculate the price and advertising equilibrium for different $\left\{\xi_{j}, \omega_{j}\right\}$ sequences, compute the derivatives at equilibrium values, and integrate out over the distribution of the $\left\{\xi_{j}, \omega_{j}\right\}$ sequences. This is computationally demanding and requires additional assumptions on the joint distribution $(\xi, \omega)$.

I form approximations to the optimal instruments, following BLP(1999), by evaluating the derivatives at the expected value of the unobservables $(\xi=\omega=0)$. The instruments will be biased since the derivatives evaluated at the expected values are not the expected value of the derivatives. However, the approximations are functions of exogenous data and are constructed such that they are highly correlated with the relevant functions of prices and advertising. Hence the exogenous instruments will be consistent estimates of the optimal instruments. ${ }^{30}$ Details are in Appendix A.

There is a potential endogeneity problem in the micro data. If a consumer with an a priori higher tendency to purchase a particular product chooses which media to consult in the decision process, then media exposure will be correlated with the unobservables. To the extent that exposure is driven by the intention to buy, exposure and purchase decisions will be correlated even if ad exposure has no impact on the purchase decision.

To account for the dependence of media exposure on the decision to buy, I would have to model the decision to engage in a particular media and define the joint probability of purchase and media exposure as a function of observables and unobservables. ${ }^{31}$ Estimation would require richer data and additional assumptions on the distribution of unobservables. I test for the exogeneity of media exposure (see Smith and Blundell, 1986; Rivers and Vuong, 1988), using purchase and media exposure data from Simmons. ${ }^{32}$ As instruments for media exposure I use the cost of access (subscription price) to various media. Details are given in Appendix B. The tests indicate media exposure endogeneity is not an issue in the data. I cannot reject the null hypothesis that exposure to newspapers, magazines, and cable television is exogenous to the PC purchase decision. Given this motivation, I treat media exposure

\footnotetext{
${ }^{30}$ One could use a series approximation (BLP) to construct exogenous instruments. I use the more direct approximation $(\mathrm{BLP}, 1999)$ since it is more closely tied to the model. Results from logit IV regressions indicate the instruments are strong and that they address the endogeneity issues.

${ }^{31}$ Anand and Shachar(2004) use micro-level data to estimate a model of TV viewing choices and show how to overcome the exposure endogeneity problem when consumption decisions also determine ad exposures.

${ }^{32}$ Rivers and Vuong (1988) develop a two-step test for the exogeneity of regressors in limited dependent variable models. Wooldridge (2002) shows the exogeneity test is valid when the regressor is a binary variable.
} 
as exogenous to the purchase decision in the structural model.

I next present an informal discussion of how variation in the data identifies the parameters. I begin with the demand side. Associated with each PC is a mean utility, which is chosen to match observed and predicted market shares. If consumers were identical, then all variation in sales would be driven by variation in product attributes. Variation in product market shares corresponding to variation in the observable attributes of those products (such as CPU speed) is used to identify the parameters of mean utility $(\beta)$.

While a PC may have attributes that are preferred by many consumers (high $\beta$ 's), it may also have attributes that appeal to certain types of consumers. For instance, if children like to play PC games, then consumers from large households may place a higher valuation on CPU speed relative to smaller households. Identification of the taste distribution parameters $(\Sigma, \Omega)$ relies on information on how consumers substitute (see 2). There are two issues that merit attention. First, new product introductions are common in the PC industry. Variation of this sort is helpful for identification of $\Sigma$. The distribution of unobserved tastes, $\nu_{i}$, is fixed over time, but the set of available products is changing over time. Variation in sales patterns over time as the set of available products change allows for identification of $\Sigma$. Second, I augment the market level data with micro data on firm choice. The extra information in the micro data allows variation in choices to mirror variation in tastes for product attributes. Correlation between $x_{j} D_{i}$ and choices identifies the $\Omega$ parameters.

If consumers were identical, then all variation in the information technology, and induced variation in shares, would be driven by variation in advertising or the age of the PC. Variation in sales corresponding to variation in PC age identifies $\vartheta$. Variation in sales corresponding to variation in advertising identifies the other parameters of $\gamma_{j}$. Returns to scale in media advertising $\left(\rho_{m}\right)$ are identified by covariation in sales with the second derivative of $a_{j m} \cdot{ }^{33}$ Identification of firm-fixed effects $\left(\Psi_{f}\right)$ is from two sources. In the macro-moments they are identified by the total variation in sales of all products sold by the firm corresponding to variation in firm advertising. In the micro-moments they are identified by observed variation in firm sales patterns corresponding to variation in firm advertising.

One major drawback of aggregate ad data is that I don't observe variation across households. Normally observed variation in market shares corresponding to variation in household ad media exposure would be necessary to identify $\Upsilon$ and $\varsigma$. The Simmons data contain useful information on media exposure across households. Variation in choices of media exposure corresponding to variation in observable consumer characteristics $\left(D_{i}^{s}\right)$ identifies $\Upsilon$. Variation in sales and ad exposure $\left(a_{j}^{\prime} \Upsilon D_{i}^{s}\right)$ identifies the effect of ad exposure on the information set $(\varsigma)$. Thus, the Simmons data allow me to side-step the need for observed ad variation

\footnotetext{
${ }^{33}$ There is not enough variation in the ad data to estimate $\varphi$ and $\rho$ effects for all media separately. I estimate these parameters for the tv medium and for the combination of newspaper and magazine media.
} 
across households. The other parameters of $\lambda_{i j}$ which do not interact with advertising $(\widetilde{\lambda})$ are separately identified from $\Omega$ due to nonlinearities. Finally, the parameters on group advertising $\left(\pi_{1}\right.$ and $\left.\pi_{2}\right)$ are identified by observed variation in expenditures on group advertisements $\left(a d_{m}\right)$ with the number of products in the group and by functional form.

Variation in prices and shares corresponding to variation in observed cost attributes identifies the corresponding cost attributes' effect on production costs. Covariation in ad prices, advertising and the generalized residuals identifies the effect of ad prices on ad costs.

\section{The Estimation Technique}

The econometric technique follows recent studies of differentiated products, such as BLP $(1995,2004)$ and Nevo $(2000)$. The parameters are $\beta, \theta=\left\{\alpha, \Sigma, \Omega, \theta_{\phi}\right\}, \eta$, and $\eta_{A D}$, where $\theta_{\phi}=\left\{\pi_{1}, \pi_{2}, \varphi, \rho, \Psi, \vartheta, \widetilde{\lambda}, \Upsilon, \varsigma\right\}$. Under the assumption that the observed data are the equilibrium outcomes, I estimate the parameters simultaneously using generalized method of moments (GMM). There are five "sets" of moments:

(i) from demand, which match the predicted market shares to observed shares

(ii) from pricing decisions, which express an orthogonality between the cost side unobservable and instruments

(iii) from advertising media decisions, which express an orthogonality between the advertising residuals and instruments

(iv) from purchase decisions, which match the model's predictions for the probability individuals purchase from firm $f$ (conditional on observed characteristics) to observed purchases

(v) from media exposure decisions, which match the model's predictions for exposure to media $m$ (conditional on observed characteristics) to observed exposure

\subsection{The Moments}

I use macro product data, ad data, and the CPS consumer data in the first three sets of moments. I use micro consumer data in the last two sets of moments. The strategy of combining micro and macro data follows work by Petrin (2002) and BLP(2004).

BLP-Type Macro Moments Following BLP, I restrict the model predictions for $j$ 's market share to match observed shares. I solve for $\delta(S, \theta)$ that is the implicit solution to

$$
S_{t}^{o b s}-s_{t}(\delta, \theta)=0
$$


where $S_{t}^{\text {obs }}$ and $s_{t}$ are vectors of observed and predicted shares respectively. I substitute $\delta(S, \theta)$ for $\delta$ when calculating the moments. ${ }^{34}$ The first moment unobservable is

$$
\xi_{j t}=\delta_{j t}(S, \theta)-x_{j}^{\prime} \beta
$$

I use the demand system estimates to compute marginal costs (Bresnahan, 1989). In vector form, the $J$ FOCs from (9) imply

$$
m c=p-\Delta(\theta, \delta)^{-1} s(\theta, \delta)
$$

where $\Delta_{j, r}=-\frac{\partial s_{r}}{\partial p_{j}} I_{j, r}$ with $I_{j, r}$ an indicator function equal to one when $j$ and $r$ are produced by the same firm. Combining (13) and (7) yields the second moment unobservable:

$$
\omega=\ln \left(p-\Delta(\theta, \delta)^{-1} s(\theta, \delta)\right)-w^{\prime} \eta
$$

Advertising Macro Moments Some firms choose not to advertise some products in some media. To allow for corner solutions I use the method of generalized residuals proposed by Gourieroux, et al.(1987). The method is best illustrated by an example. For ease of exposition I suppress the time subscript. Let $y_{i}^{*}=x_{i} \beta+u_{i}$. We observe $y_{i}^{*}$ if $y_{i}^{*} \geq 0$ and zero otherwise. The errors, $u_{i}(\beta)$, are linked with $y_{i}^{*}$. The errors cannot be used to construct moments because they depend on unobserved variables. Gourieroux, et al. suggest an alternative method: replace the errors by their best prediction conditional on the observable variables, $E\left[u_{i}(\beta) \mid y_{i}\right]$, and use these to construct moments.

In this paper the latent variables are optimal advertising levels (denoted $a_{j m}^{*}$ ). Due to nonlinearities the application is more complex, but the technique is the same. We observe

$$
a_{j m}=\left\{\begin{aligned}
a_{j m}^{*} & \text { if } \partial \Pi_{j} /\left.\partial a_{j m}\right|_{a_{j m}=a_{j m}^{*}}=0 \\
0 & \text { if } \partial \Pi_{j} /\left.\partial a_{j m}\right|_{a_{j m}=0}<0
\end{aligned}\right.
$$

where $\Pi_{j}$ is product $j$ 's profit from (6). Rewrite the advertising medium FOC as

$$
\ln \left(m r_{j m}\left(a_{j m}\right)\right)-w_{j m}^{a d \prime} \psi=\tau_{j m}
$$

where $m r_{j m}$ is medium marginal revenue (the left-hand side of (10)). The latent variable is the implicit solution to (15) so the errors, $\tau_{j m}$, will depend on $a_{j m}^{*}$. I use the best prediction of $\tau_{j m}$, conditional on observed advertising, to construct moments. In estimation, I fix $\sigma_{\tau}=1$.

Using ad marginal costs (8) and the interior FOCs (10), the likelihood function is

$$
£=\prod_{j: a_{j m}>0} \phi_{\text {normal }}\left(\widetilde{m r}_{j m}\right) \prod_{j: a_{j m} \leq 0} 1-\Phi\left(\widetilde{m r}_{j m}\right)
$$

\footnotetext{
${ }^{34}$ I use a contraction mapping suggested by BLP to compute $\delta(S, \theta)$. Goeree (2008) shows that the function used in the fixed point algorithm is a contraction mapping. The proof parallels the proof for the full information case.
} 
where $\widetilde{m r}_{j m} \equiv \ln \left(m r_{j m}\left(a_{j m}\right)\right)-w_{j m}^{a d \prime} \psi, \phi_{\text {normal }}$ is the standard normal pdf, and $\Phi$ is the cumulative standard normal. The generalized residual for the $j$ th observation is

$$
\widetilde{\tau}_{j m}(\widehat{\Xi})=E\left[\tau_{j m}(\widehat{\Xi}) \mid a_{j m}\right]=\widetilde{m r}_{j m} 1\left(a_{j m}>0\right)-\frac{\phi_{\text {normal }}\left(\widetilde{m r}_{j m}\right)}{1-\Phi\left(\widetilde{m r}_{j m}\right)} 1\left(a_{j m}=0\right)
$$

where $\Xi$ are the parameters of (15) and $\widehat{\Xi}$ its maximum likelihood estimator.

The (third set of) moments express an orthogonality between the generalized residuals and the instruments. For instance, the $\Xi$ that solves

$$
\frac{1}{J} \sum_{j} \frac{\partial \widetilde{m r}}{\partial \Xi} \widetilde{\tau}_{j m}=0
$$

is the MOM estimator, where $\frac{\partial \widetilde{m} r_{j m}}{\partial \Xi}$ are the appropriate instruments. Let $\mathcal{T}\left(\delta, m c, \theta, \eta_{\mathrm{AD}}\right)$ be the vector of residuals stacked over media and products.

Firm Choice Micro Moments I combine micro firm choice data from Simmons with macro product level data (á la Petrin, 2002). ${ }^{35}$ The Simmons data connect consumers to firms, thus associating consumer and average product attributes (across firms). These moments allow me to obtain more precise estimates of the parameters of the taste distribution $(\Omega$ and $\Sigma)$ and advertising effectiveness $\left(\Psi_{f}\right)$. The demographic characteristics for these moments (denoted $D^{s}$ ) are not given by the CPS but are linked directly to purchases.

Let $B_{i}$ be a $F \times 1$ vector of firm choices for individual $i$. Let $b_{i}$ be a realization of $B_{i}$ where $b_{i f}=1$ if a brand produced by $f$ was chosen. Define the residual as the difference between the vector of observed choices and the model prediction given $(\delta, \theta)$ :

$$
\mathcal{B}_{i}(\delta, \theta)=b_{i}-E_{\nu, \kappa} E\left[B_{i} \mid D_{i}^{s}, \delta, \theta\right]
$$

For example, the element of $E_{\nu, \kappa} E\left[B_{i} \mid D_{i}^{s}, \delta, \theta\right]$ corresponding to firm 2 for consumer $i$ is

$$
\sum_{j \in \mathcal{J}_{2}} \int \sum_{\mathcal{S} \in \mathcal{C}_{j}} \prod_{l \in \mathcal{S}} \phi_{i l t} \prod_{k \notin \mathcal{S}}\left(1-\phi_{i k t}\right) \frac{\exp \left\{\delta_{j t}+\mu_{i j t}\right\}}{y_{i t}^{\alpha}+\sum_{r \in \mathcal{S}} \exp \left\{\delta_{r t}+\mu_{i r t}\right\}} d G_{\nu}(\nu) d G_{\kappa}(\kappa)
$$

where the first summand is over products sold by firm 2, the integral is over the assumed distributions of $\nu$ and $\kappa$, and the second summand is over all the different choice sets that include product $j .{ }^{36}$ The population restriction for the micro moment is $E\left[\mathcal{B}_{i}(\delta, \theta) \mid(x, \xi)\right]=$ 0 . Let $\mathcal{B}(\delta, \theta)$ be the vector formed by stacking the residuals $\mathcal{B}_{i}(\delta, \theta)$ over individuals.

\footnotetext{
${ }^{35}$ Petrin (2002) shows how to combine macro data with data that links average consumer attributes to product attributes to obtain more precise estimates.

${ }^{36}$ Simmons is annual so the outermost summand is over all products sold by each firm over the year.
} 
Media Exposure Micro Moments The fifth set of moments are used to estimate $\Upsilon$. These allow me to control for variation in ad exposure across households (as related to observables) via variation in media exposure. The Simmons respondents were ranked according to how often they watched TV, read newspapers, etc. relative to others in the surveyed population. I have information on the ranges of respondents' answers, but the survey reports only the quintile to which the consumer belongs. I construct moments arising from an ordered-response likelihood. Let $h_{i m}^{*}$ be the amount of exposure of $i$ to medium $m$

$$
h_{i m}^{*}=D_{i}^{s \prime} \Upsilon_{m}+\varepsilon_{i m}
$$

where $\varepsilon_{i m}$ is a mean zero term distributed i.i.d. standard normal. Defining quintile one as the highest, $i$ belongs to the $q$ th quintile in medium $m$ if $c_{q m}<h_{i m}^{*}<c_{(q-1) m}$ where $c$ are cutoff values. Let $H_{i m}$ be the vector of quintiles for $i$ in $m$. Let $h_{i m}$ be a realization of $H_{i m}$ where the $q$ th element $h_{i m q}=1$ if $i$ 's level of exposure falls in $q$. If $\Phi$ is the cumulative standard normal and $\Phi_{i q m}=\Phi\left(c_{q m}-D_{i}^{s \prime} \Upsilon_{m}\right)$ then

$$
\operatorname{Pr}\left(h_{i q m}=1\right)=\Phi_{i, q-1, m}-\Phi_{i q m} .
$$

The maximum likelihood estimate of $\Upsilon_{m}$ solves

$$
\sum_{i} \sum_{q} h_{i q m} \frac{\partial \ln \operatorname{Pr}\left(h_{i q m}=1 \mid D_{i}^{s}\right)}{\partial \Upsilon_{m}}=0 .
$$

The difference between the vector of observed quintiles and the prediction given $\Upsilon_{m}$,

$$
\mathcal{H}_{i m}\left(\Upsilon_{m}\right)=h_{i m}-E\left[H_{i m} \mid D_{i}^{s}, \Upsilon_{m}\right],
$$

is the residual where the $q$ th element of $E\left[H_{i m} \mid D_{i}^{s}, \Upsilon_{m}\right]=\Phi_{i q m}-\Phi_{i, q-1, m}$ and

$$
Z_{\text {media }, i m}=\frac{\partial \ln \operatorname{Pr}\left(h_{i q m}=1 \mid D_{i}^{s}\right)}{\partial \Upsilon_{m d}}
$$

are the appropriate instruments. Let $\mathcal{H}_{i}(\Upsilon)$ be the residuals stacked over media.

\subsection{The GMM Estimator}

I use GMM to find the parameter values that minimize the objective function, $\Lambda^{\prime} Z A^{-1} Z^{\prime} \Lambda$, where $A$ is a weighting matrix, which is a consistent estimate of $E\left[Z^{\prime} \Lambda \Lambda^{\prime} Z\right]$ and $Z$ are instruments orthogonal to the composite error term $\Lambda$. Specifically, if $Z_{\xi}, Z_{\omega}, Z_{a d}, Z_{\text {micro }}$, $Z_{\text {media }}$ are the respective instruments for each disturbance/residual, the sample moments are

$$
Z^{\prime} \Lambda=\left[\begin{array}{c}
\frac{1}{J} \sum_{j=1}^{J} Z_{\xi, j} \xi_{j}(\delta, \beta) \\
\frac{1}{J} \sum_{j=1}^{J} Z_{\omega, j} \omega_{j}(\delta, \theta, \eta) \\
\frac{1}{J} \sum_{j=1}^{m * J} Z_{a d, j} \mathcal{T}_{j}\left(\delta, \theta, \eta_{A D}\right) \\
\frac{1}{N} \sum_{i=1}^{N} Z_{\text {micro, } i} \mathcal{B}_{i}(\delta, \theta) \\
\frac{1}{N} \sum_{i=1}^{N} Z_{\text {media }, i} \mathcal{H}_{i}(\Upsilon)
\end{array}\right]
$$


where $Z_{\xi, j}$ is column $j$ of $Z_{\xi}$. Joint estimation takes into account the cross-equation restrictions on the parameters that affect both demand and supply, which yields more efficient estimates. This comes at the cost of increased computation time since joint estimation requires a non-linear search over all the parameters of the model. ${ }^{37}$

Simulation As in BLP, the distribution of consumer demographics is an empirical one. As a result there is no analytical solution for predicted market shares, making simulation of equation (5) necessary. Furthermore consumers may not know all products for sale, but I don't observe the choice set facing any one consumer. As I discussed in section 3.2, a solution is to simulate the choice set. ${ }^{38}$ An outline of the simulation technique follows. Details are in Appendix C.

I sample a set of "individuals" where each consists of $\left(v_{i 1}, \ldots, v_{i k}\right)$ taste parameters drawn from a multivariate normal; demographic characteristics, $\left(y_{i}, D_{i 1}, \ldots, D_{i d}\right)$, drawn from the CPS for use in the macro moments; and unobserved advertising medium effectiveness draws, $\left(\kappa_{i 1}, \ldots, \kappa_{i m}\right)$, from a multivariate log normal.

Simulating individual $i$ 's choice set is a two-step process. I begin by drawing $J$ uniform variables for each individual. First, I compute the probability individual $i$ knows product $j$ for a given value of the parameters. That is I compute the information technology for each person-product combination (the $\phi_{i j}$ from equation (4) evaluated at the parameter values). Second, I compare $i$ 's uniform draw for each product with the computed $\phi_{i j}$. If the computed probability $i$ knows product $k$ (ie. the value of $\phi_{i k}$ ) is larger than the corresponding uniform draw for $k$, product $k$ is in $i$ 's choice set. I repeat this comparison for all products and form $i$ 's simulated choice set. Note that $i$ 's choice set may change as the parameter values change. I simulate the choice set for the remaining individuals analogously.

Given the simulated choice set, I compute choice probabilities for each individual for each product and construct an importance sampler to smooth the simulated choice proba-

\footnotetext{
${ }^{37}$ I restrict the non-linear search to a subset of the parameters $\Omega=\left\{\theta, \eta_{A D}\right\}$. This restriction is possible since the FOCs with respect to $\beta$ and $\eta$ can be expressed in terms of $\theta$. (See Nevo, 2000.) I could separately estimate $\Upsilon$ and substitute predicted for actual exposure when estimating the remaining parameters. This would decrease computational time but, due to the non-linear nature of the model, would not yield consistent estimates except under specific distributional assumptions.

${ }^{38}$ Chiang, et al.(1999) use micro purchase data for ketchup to model "consideration set" formation. A consideration set is a subset of the $2^{J-1}$ choice sets. Due to the stable nature of the industry the consumer's consideration set doesn't change over time, allowing the authors to eliminate choice sets which do not contain all previously purchased brands. Also, there are only four brands for a consumer to consider. The PC industry is much different: it is rapidly changing and there are a large number of products. Therefore, I use a very different approach in modeling (and estimating) choice set heterogeneity. While the approach I take does not a priori limit the potential set of products available to the consumer, the Chiang, et al. approach is more flexible in the sense that it does not impose conditional independence among products in a particular consumer's consideration set. Recent papers addressing consideration sets are Mehta, et al.(2003), Nierop, et al.(2005), and Ching, et al.(2007).
} 
bilities. ${ }^{39}$ The market share simulator is the average over individuals of the smoothed choice probabilities. The process is similar for the micro moments, but I take $R$ draws for each product-individual. The individual product choice probability simulator is the average over the $R$ draws. Individual firm choice probabilities are the sum over the products offered by each firm.

The Estimation Algorithm and Properties of the Estimator First, calculate the instruments and keep them fixed for the duration of the estimation. Then, given a value of the parameters, $\Theta$,

(i) Compute the simulated market shares and solve for the vector $\delta$ that equates simulated and observed shares.

(ii) Calculate $\beta$ and compute the demand unobservables, $\xi$ (see 12). Calculate $\eta$ and compute the cost side unobservables, $\omega$ (see 14). Compute the ad residual, $\mathcal{T}$.

(iii) Simulate the firm purchase probabilities and calculate the micro residual (see 17).

(iv) Compute the media residual (see 18).

(v) Search for the parameter values that minimize the objective function: $\widehat{\Lambda}^{\prime} Z A^{-1} Z^{\prime} \widehat{\Lambda}$, where $\widehat{\Lambda}$ is the composite error term resulting from simulated moments. If the parameters don't minimize the moments (according to some criteria) make a new guess of the parameters. Repeat until moments are close to zero.

The estimator is consistent and asymptotically normal (Pakes and Pollard, 1989). As the number of pseudo random draws used in simulation $R \rightarrow \infty$ the method of simulated moments covariance matrix approaches the method of moments covariance matrix. To reduce the variance due to simulation, I employ antithetic acceleration (see Stern, 1997, 2000). Geweke (1988) shows if antithetic acceleration is implemented during simulation, then the loss in precision is of order $1 / N$ (where $N$ are the number of observations), which requires no adjustment to the asymptotic covariance matrix. The reported (asymptotic) standard errors are derived from the inverse of the simulated information matrix which allows for possible heteroskedasticity. ${ }^{40}$

\footnotetext{
${ }^{39}$ I construct an importance sampler by using the initial choice set weight to smooth the simulated choice probabilities. The initial choice set weight is the product over the $\phi$ 's for products in the choice set (computed at initial parameter values) multiplied by the product of $(1-\phi)$ for all products not in the choice set.

${ }^{40}$ The reported standard errors do not include additional variance due to simulation error.
} 


\section{Preliminary Analysis}

First, I estimate a series of probit models of the decision to purchase a PC (using the Simmons data). ${ }^{41}$ These regressions establish that advertising exposure impacts demand and guide the choice of variables to include in the structural model. I started by allowing for many explanatory variables including interactions between consumer attributes, education and income splines, and media exposure variables (see Appendix D, Table D1 for selected results). The estimates suggest media exposure affects the decision to buy a PC, after controlling for observed consumer covariates. ${ }^{42}$ Results from likelihood ratio tests reject the hypothesis that media exposure has no effect on PC purchase (at 1\% significance level) and indicate exposure to the TV and magazine media impact the purchase decision the most. ${ }^{43}$ I found the consumer attributes which matter most are age, education, and marital status. Household income and size also significantly affect the probability of purchase, although including the presence and/or number of kids does not improve the fit.

Next, I estimate models of firm choice that illustrate the need to instrument for price and advertising in the structural model. As discussed in section 4, advertising may be endogenous. Due to data limitations I cannot examine the effects of product advertising on product choice without estimating the structural model. Instead, I examine the effects of firm advertising on firm choice using Simmons data and CMR advertising data combined with data on observable product characteristics. Suppose a consumer who buys a computer first chooses a firm and then a model. Let the consumer's indirect utility be a function of observed attributes that vary by model and firm (these are price, CPU speed, form factor etc.), of observed attributes that vary only by firm (these are firm advertising), and a generalized extreme value term. Table D2 in Appendix D presents results of the nested logit regresssions.

In all specifications price coefficient estimates are positive and significant. The most obvious explanation is that prices are correlated with quality. After including CPU speed, Pentium, and laptop as explanatory variables (specification 2), the price coefficient is still positive suggesting there are other product attributes that are positively correlated with prices. Specification 3, which includes total advertising expenditures as an explanatory variable, fits better even though it has fewer explanatory variables. Without indicating

\footnotetext{
${ }^{41}$ While reduced form estimation is computationally easy, structural analysis has many advantages. It provides estimates that are invariant to changes in policy or competitive factors. It also allows one to specify the effects of advertising. If advertising affects a consumer's choice set we would expect changes in behavior as advertising changes. This effect is not captured in reduced form models because it is not possible to be specific about how advertising affects demand. Also we would expect changes in firm behavior as variables relating to advertising change, which will have an impact on markups and prices.

${ }^{42}$ Unobserved consumer attributes may influence media effectiveness at providing information. The full model allows for unobserved consumer heterogeneity in media effectiveness (the $\kappa_{i}$, see section 3.2).

${ }^{43}$ I cannot reject the hypothesis that all other media have no impact on purchase probabilities.
} 
how advertising affects demand the coefficient estimates indicate that advertising may be correlated with higher quality. This obtains from comparing estimates from specifications 1 and 3: price coefficients in the specification with advertising are smaller. Advertising may be capturing some of the effect of unobserved product attributes. ${ }^{44}$ The results suggest advertising's effect differs across media (specification 4). Finally, after including consumer covariates (specification 6), advertising still influences the decision of firm choice.

I account for the possibility that unobserved attributes are correlated with prices and correct for the possible correlation with advertising in the structural model. Previous papers (Berry, 1994; BLP, 1995, 1999; Nevo, 2000; and many others) have shown that BLP-type instruments (which I use) can account for the possible correlation between prices and unobserved characteristics and result in a more reasonable estimate of the coefficient on price.

Finally, I estimate a logit model to show that the instruments I use in the full model address the endogeneity issues. Table D3 in Appendix D presents results. As previous studies have shown, logit demand estimates are obtained from an ordinary least squares (OLS) regression of $\ln \left(s_{j}\right)-\ln \left(s_{0}\right)$ on price, other product characteristics, and firm dummy variables. Included product characteristics are the same as those in the full specification. The first two columns report OLS results. As expected, the price coefficient is negative but small in magnitude. The second column reports results with firm dummy variables, which improves the fit of the model, but does not significantly change the price coefficient estimate. Columns (iii)-(iv) present results using BLP (1995) instruments. These instruments are the sum of the values of the same characteristics of other products offered by the same firm, the sum of the values of the same characteristics of all products offered by rival firms, and the number of own-firm products and number of rival firm products. The remaining columns present the results from instrumental variables (IV) regressions using a more direct (but computationally burdensome) approximation to the efficient IV estimator in the spirit of BLP (1999). See Appendix A for details.

Both sets of instruments appear to address the endogeneity of price issue and result in estimates for the price coefficient that is significantly higher in absolute value. Other parameter estimates are similar across specifications, with an exception being the sign change on the coefficient for laptop. This is consistent with the idea that price is endogenous as laptops are more portable and hence better (all else constant) and certainly demand a higher price. The first-stage F-statistic for the IV regressions are high suggesting the instruments have power. While the results suggest both sets of instruments are reasonable candidates to use in the full-model, I chose to use the more direct approximation to the optimal instruments

\footnotetext{
${ }^{44}$ Comparing specifications 2 and 5 suggests that advertising may impact choice as much as observable product characteristics. However these results should be interpreted with caution since the coefficients on product characteristics are estimable up to a scale factor and are identified due to nonlinearities.
} 
(based on BLP, 1999) since they are more closely tied to the structure of the model.

\section{Structural Estimation Results}

Product Differentiation There is much variation in tastes across consumers with respect to product attributes. I estimate the means and the standard deviations of the taste distribution for CPU speed, Pentium, and laptop. In all tables the (asymptotic) standard errors are in parentheses. The mean coefficients $(\beta)$ are given in the first column and panel of Table III. Estimates of heterogeneity around these means are presented in the next columns. The means of CPU speed and laptop are positive and significant. The results imply that CPU speed and laptop have a significant positive effect on the distribution of utility. In addition, the marginal valuation for CPU speed is (significantly) increasing in household size (4.05). This is intuitive as children often use the PC to play games (which require higher CPU speeds). Coefficients for Pentium dummy are not significant at the $5 \%$ level. This suggests that once you control for CPU speed (and other product attributes) consumers don't place extra value on whether the chip is a Pentium. During this time period $80 \%$ of PCs had a Pentium chip. In that light the results may not be so surprising.

The non-random coefficient results are also presented in the first panel. The coefficient on $\ln (y-p)$ is of the expected sign and is highly significant (1.2). Firm fixed effect estimates indicate that the marginal valuation for a product is (significantly) higher if it is produced by Apple, Dell, IBM or Packard Bell. This could capture prestige-effects of owning a computer produced by one of top firms (Apple, IBM, and Packard Bell). Apple operates on a different platform, so Apple fixed effects could reflect the extra valuation consumers, on average, place on the Apple platform. Finally they could capture extra valuation consumers place on enhanced services offered by the firms (for instance Dell is known for its excellent consumer service) or other reputational effects.

The cost and non-home sector estimates are given in the lower panel. Most of the coefficients $(\eta)$ are of the expected sign and are significantly different from zero. The estimates indicate marginal costs are declining over time and increases in CPU speed or producing a laptop increase marginal costs. The only variable with an unexpected sign is Pentium (-0.25), indicating that PCs with a Pentium chip are cheaper to produce. The coefficient on the $(\log )$ price of advertising $(\psi)$ is highly significant and indicates that there are not many product-specific cost characteristics that affect the cost of advertising.

The parameter estimates for non-home marginal revenue are given in the bottom panel. All coefficients are positive and significant. Recall the majority of industry advertising expenditures are by IBM. My conjecture that the high expenditures are due to IBM's non- 
PC enterprises seems to be supported. I included non-PC sales in the non-home marginal revenue to adjust for the fact that the measure of advertising includes some for non-PCs. The coefficient on non-PC sales (3.7) is significant (at the $5 \%$ level) and positive. But the interaction term between IBM and advertising in the information technology (0.9) indicates that advertising by IBM is still more effective relative to some other firms, after controlling for non-PC enterprises. If the IBM fixed effect in the information technology were not significantly different from zero then I would have concluded that the presence of IBM in the non-PC sector fully explained their large advertising expenditures.

\begin{tabular}{|c|c|c|c|c|c|c|c|c|}
\hline \multirow[t]{2}{*}{ Variable } & \multirow[b]{2}{*}{ Coefficient } & \multirow{3}{*}{$\begin{array}{c}\text { Std } \\
\text { Error } \\
\end{array}$} & \multirow[b]{2}{*}{$\begin{array}{l}\text { Standard } \\
\text { Deviation }\end{array}$} & \multirow[b]{2}{*}{$\begin{array}{c}\text { Std } \\
\text { Error }\end{array}$} & \multicolumn{4}{|c|}{ Interactions with Demographics } \\
\hline & & & & & $\begin{array}{l}\text { household } \\
\text { size }\end{array}$ & $\begin{array}{l}\text { income }> \\
\$ 100,000\end{array}$ & $\begin{array}{c}\text { age } 30 \\
\text { to } 50\end{array}$ & $\begin{array}{l}\text { white } \\
\text { male }\end{array}$ \\
\hline \multicolumn{8}{|l|}{ utility coefficients } & \\
\hline constant & -12.026 ** & $(0.796)$ & 0.044 & $(0.558)$ & & & & \\
\hline cpu speed (MHz) & 9.288 ** & $(1.599)$ & 0.156 ** & $(0.017)$ & $\begin{array}{l}4.049 \\
(0.674)\end{array}$ & & & \\
\hline pentium & 1.236 * & $(0.890)$ & 0.209 & $(0.886)$ & & $\begin{array}{c}0.016 \\
(0.489)\end{array}$ & & \\
\hline laptop & 2.974 ** & $(0.525)$ & 0.953 & $(4.619)$ & & & $\begin{array}{c}2.048 \\
(8.870)\end{array}$ & $\begin{array}{r}4.099 \\
(9.192)\end{array}$ \\
\hline In(income-price) & $1.211 * *$ & $(0.057)$ & & & & & & \\
\hline acer & 2.624 & $(4.900)$ & & & & & & \\
\hline apple & $3.070^{* *}$ & $(1.032)$ & & & & & & \\
\hline compaq & 2.662 & (18.009) & & & & & & \\
\hline dell & 2.658 ** & $(0.301)$ & & & & & & \\
\hline gateway & 7.411 & (14.615) & & & & & & \\
\hline hewlett packard & 1.309 & $(3.905)$ & & & & & & \\
\hline ibm & $2.514^{* *}$ & $(0.712)$ & & & & & & \\
\hline micron & -1.159 & $(6.011)$ & & & & & & \\
\hline packard bell & 4.372 & $(4.002)$ & & & & & & \\
\hline \multicolumn{9}{|c|}{ cost side parameters } \\
\hline \multicolumn{9}{|c|}{ In marginal cost of production } \\
\hline constant & $7.427^{* *}$ & $(0.212)$ & & & & & & \\
\hline In(cpu speed) & 0.462 ** & $(0.044)$ & & & & & & \\
\hline pentium & $-0.250 * *$ & $(0.007)$ & & & & & & \\
\hline laptop & 1.204 ** & $(0.071)$ & & & & & & \\
\hline quarterly trend & -0.156 ** & $(0.027)$ & & & & & & \\
\hline \multicolumn{9}{|c|}{ In marginal cost of advertising } \\
\hline constant & 2.631 & $(7.087)$ & & & & & & \\
\hline price of advertising & $1.051^{* *}$ & $(0.074)$ & & & & & & \\
\hline \multicolumn{9}{|c|}{ non-home sector marginal revenue } \\
\hline constant & 11.085 & (278.374) & & & & & & \\
\hline non-home sector price & $1.815^{* *}$ & $(0.354)$ & & & & & & \\
\hline cpu speed & $0.010^{* *}$ & $(0.004)$ & & & & & & \\
\hline non-pc sales & $3.688^{*}$ & $(1.881)$ & & & & & & \\
\hline
\end{tabular}

Table III: Structural Estimates of Utility and Cost Parameters

Consumer Information Heterogeneity and Advertising Effectiveness Not surprisingly the results indicate that advertising has very different effects across individuals and that exposure to advertising significantly impacts the information set. The first panel of Table IV presents estimates of how media exposure varies with observed demographic characteristics $(\Upsilon)$. These coefficients proxy for effectiveness of ads in reaching consumers through 
various media. The results indicate magazines are most effective at reaching high income individuals where the effectiveness is increasing in household size. Newspapers are most effective at reaching high income, married individuals who are above the age of 30 . Although newspaper advertising is less likely to reach a family the larger is their household (-0.04). Hence, newspaper advertising targeted at large households would not be effective in increasing the probability of being informed for this particular cohort. Perhaps not surprisingly, TV advertising is the most effective medium for reaching low income households. Television advertising is also effective at reaching married individuals over 50, although not as effective as newspaper. Interestingly most advertising in the PC industry is in magazines, suggesting PC firms target high income households.

\begin{tabular}{|c|c|c|c|c|c|c|c|c|c|c|c|}
\hline \multirow[b]{3}{*}{ Variable } & \multirow{3}{*}{\multicolumn{2}{|c|}{ Coefficient }} & & \multicolumn{8}{|c|}{ Coefficient estimates for interactions with media } \\
\hline & & & & \multicolumn{2}{|c|}{ Magazine (mag) } & \multicolumn{2}{|c|}{ Newspaper (np) } & \multicolumn{2}{|c|}{ Television (tv) } & \multicolumn{2}{|c|}{ Radio } \\
\hline & & & Std. Error & Coefficient & Std. Error & Coefficient & Std. Error & Coefficient & Std. Error & Coefficient & Std. Error \\
\hline \multicolumn{12}{|c|}{ consumer information heterogeneity coefficients } \\
\hline \multicolumn{12}{|c|}{ media and demographic interactions $(\mathrm{Y})$} \\
\hline constant & & & & $-1.032 * *$ & $(0.040)$ & $-0.973 * *$ & $(0.040)$ & $-1.032 * *$ & $(0.041)$ & $-1.000 * *$ & $(0.043)$ \\
\hline 30 to 50 ( $=1$ if $30<$ age $<50$ & & & & $-0.042 *$ & $(0.025)$ & $0.207^{* *}$ & $(0.025)$ & 0.019 & $(0.025)$ & $-0.030 *$ & $(0.025)$ \\
\hline 50 plus $(=1$ if age $>50)$ & & & & 0.005 & $(0.025)$ & $0.541^{\star *}$ & $(0.025)$ & 0.193 ** & $(0.025)$ & $-0.245 * *$ & $(0.025)$ \\
\hline married ( $=1$ if married) & & & & $-0.022 *$ & $(0.018)$ & $0.187^{* *}$ & $(0.018)$ & 0.075 ** & $(0.018)$ & -0.011 & $(0.018)$ \\
\hline hh size (household size) & & & & $0.040 * *$ & $(0.006)$ & $-0.038^{* *}$ & $(0.006)$ & $0.018^{* *}$ & $(0.006)$ & 0.012 * & $(0.006)$ \\
\hline inclow ( $=1$ if income $<\$ 60,000)$ & & & & $-0.194 * *$ & $(0.021)$ & $-0.251 * *$ & $(0.021)$ & $0.114^{* *}$ & $(0.021)$ & $-0.117^{* *}$ & $(0.022)$ \\
\hline inchigh $(=1$ if income $>\$ 100,00$ & & & & 0.153 ** & $(0.029)$ & $0.127^{\star *}$ & $(0.028)$ & -0.025 & $(0.030)$ & 0.069 ** & $(0.030)$ \\
\hline malewh ( $=1$ if male and white) & & & & $-0.078^{* *}$ & $(0.018)$ & 0.002 & $(0.018)$ & $-0.019 *$ & $(0.018)$ & 0.006 & $(0.018)$ \\
\hline eduhs ( $=1$ if highest edu 12 ye & ars) & & & $-0.102 * *$ & $(0.026)$ & $-0.338 * *$ & $(0.026)$ & 0.296 ** & $(0.027)$ & 0.076 ** & $(0.027)$ \\
\hline eduad $(=1$ if highest edu $1-3 \mathrm{c}$ & ollege) & & & $0.032 *$ & $(0.028)$ & $-0.166^{* *}$ & $(0.027)$ & $0.278^{* *}$ & $(0.028)$ & $0.115^{* *}$ & $(0.029)$ \\
\hline edubs ( $=1$ if highest edu colleg & ye grad) & & & -0.024 & $(0.025)$ & -0.063 ** & $(0.024)$ & $0.145^{* *}$ & $(0.025)$ & 0.081 ** & $(0.026)$ \\
\hline edusp (education if $<11$ ) & & & & -0.028 ** & $(0.003)$ & -0.069 ** & $(0.003)$ & 0.034 ** & $(0.003)$ & $-0.014^{* *}$ & $(0.003)$ \\
\hline advertising media exposure $(\zeta)$ & & & & & & & & & & & \\
\hline $\begin{array}{l}\text { media exposure * advertising } \\
\text { demographics }(\lambda)\end{array}$ & 0.948 & & $(0.059)$ & & & & & & & & \\
\hline constant & 0.104 & ** & $(0.004)$ & & & & & & & & \\
\hline high school graduate & 0.834 & ** & $(0.028)$ & & & & & & & & \\
\hline income $<\$ 60,000$ & 0.687 & ** & $(0.009)$ & & & & & & & & \\
\hline income $>\$ 100,000$ & 0.139 & & $(0.318)$ & & & & & & & & \\
\hline \multicolumn{12}{|c|}{ information technology coefficients common across consumers } \\
\hline $\begin{array}{l}\text { age of pc } \\
\text { media advertising }(\varphi, \rho)\end{array}$ & 0.159 & ** & $(0.005)$ & & & & & & & & \\
\hline npand mag advertising & 0.720 & * & $(0.488)$ & & & & & & & & \\
\hline tv advertising & 1.078 & ** & $(0.418)$ & & & & & & & & \\
\hline (np and mag advertising $)^{2}$ & -0.013 & & $(0.014)$ & & & & & & & & \\
\hline (tv advertising $)^{2}$ & -0.049 & ** & $(0.004)$ & & & & & & & & \\
\hline \multicolumn{12}{|l|}{ firm total advertising $(\Psi)$} \\
\hline acer & 0.520 & & $(0.042)$ & & & & & & & & \\
\hline apple & 0.163 & & $(0.790)$ & & & & & & & & \\
\hline compaq & 0.504 & ** & $(0.077)$ & & & & & & & & \\
\hline dell & 0.497 & * & $(0.460)$ & & & & & & & & \\
\hline gateway & 0.918 & ** & $(0.065)$ & & & & & & & & \\
\hline hewlett packard & 0.199 & & $(11.750)$ & & & & & & & & \\
\hline $\mathrm{ibm}$ & 0.926 & ** & $(0.184)$ & & & & & & & & \\
\hline micron & 0.029 & & (5.832) & & & & & & & & \\
\hline packard bell & 0.231 & * & $(0.149)$ & & & & & & & & \\
\hline \multicolumn{12}{|l|}{ group advertising $(\pi)$} \\
\hline group advertising & 0.891 & $\star \star$ & $(0.007)$ & & & & & & & & \\
\hline (group advertising) $^{2}$ & $0.104 *$ & ** & $(0.011)$ & & & & & & & & \\
\hline
\end{tabular}

Table IV: Structural Estimates of Information Technology Parameters

The results confirm that variation in ad media exposure across households is an important source of consumer heterogeneity. The variation in ad exposure translates into variation in information sets as evidenced by the positive and highly significant estimate for $\varsigma$. The 
estimates highlight the importance of considering the differential effects of advertising both across households and across media. Most of the literature does not incorporate consumer information heterogeneity, which has implications for markups as discussed shortly.

Parameter estimates of $\widetilde{\lambda}$ suggest other means of information provision, such as word-ofmouth or experience, play a role in informing certain types of consumers. The coefficient on income less than $\$ 60,000$ (0.69) indicates these individuals are likely to be informed about $41 \%$ of the products without seeing an ad. Whereas having a high income is not significantly different from having a middle income, in terms of being informed without seeing an ad. This could arise because low income individuals are likely to have lower opportunity costs and thus more time to search for information. In addition, the probability of being informed without seeing any advertising is higher for high-school grads relative to non-graduates.

The lower panel presents estimates of the parameters that are the same across households (the $\gamma_{j}$ parameters). Consumers are significantly more likely to know a PC the longer it has been on the market (0.16). This is intuitive, for the longer it has been on the market the more opportunity consumers have had to learn of it by word-of-mouth or through advertising. There are decreasing returns to advertising in the TV (-0.05) and newspapers and magazines $(-0.01)$ media, but they are decreasing at a faster rate for TV. Estimates of firm fixed effects interacted with total advertising $(\Psi)$ indicate that some firms are more effective at informing consumers through advertising. Most notably ads by Compaq, Dell, Gateway, IBM and Packard Bell are significantly more effective, which could be due to differences in advertising techniques across firms.

Some products are advertised in groups while others are advertised individually. The coefficient estimates on group advertising $\left(\pi_{1}\right)$ and group advertising squared $\left(\pi_{2}\right)$ are given in the last rows of Table IV. These (unrestricted) estimates predict that we will observe both group and product specific advertising, which is supported by the data. There are economies of scope in group advertising (0.1). The estimates imply that if average group ad expenditures $(\overline{a d})$ for a particular product group are above a threshold level of $\$ 1.05$ million per quarter ${ }^{45}$ (either the expenditures for a group are high or the groups are small) the firm will find it worthwhile to engage in group advertising to capitalize on the economies of scope. To put this into context, in the first quarter of 1998 Apple's advertising strategy involved 17 group advertisements. The estimates suggest we would observe 17 group ads only if Apple's home sector advertising budget was at least $\$ 18$ million. Apple spent over $\$ 180$ million in advertising in 1998 and more than $\$ 20$ million in the first quarter - consistent with the model's prediction.

\footnotetext{
${ }^{45}$ The ad threshold is $\left(1-\pi_{1}\right) / \pi_{2}$. If there is only one product in the group I restrict $\pi_{1}=1$ and $\pi_{2}=0$.
} 
Substitution Patterns and Information Provision The estimated parameters have important implications for pricing and advertising behavior and markups. The markups earned by firms are determined, in part, by the substitution behavior of consumers. Substitution could be induced by changes in prices or choice sets, the latter of which is significantly impacted by advertising with varying effects across consumers. When advertising changes the impact on the choice set is more pronounced for those consumers who are more sensitive to advertising. The firms decisions of what prices to charge and how much information to provide through advertising depend upon the price and advertising elasticities of demand.

The top panel of Table V presents a sample from 1998 of own- and cross-price elasticities of demand. ${ }^{46}$ The table shows all negative elements on the diagonal. Consistent with oligopolistic conduct, the results indicate that the products are priced in the elastic portion of the demand curve. The results show that products are more sensitive to changes in prices of computers with similar characteristics. For example, Apple computers are most sensitive to changes in the prices of other Apple computers implying there is less substitution across platforms. Among PC's that have a windows operating system, form factor plays a strong role in substitution patterns. For example, Compaq Armada laptop is most sensitive to changes in prices of other laptops rather than to changes in other Compaq non-laptop computers. These intuitive substitution patterns are consistent across the data.

\begin{tabular}{|c|c|c|c|c|c|c|c|c|c|}
\hline & $\begin{array}{c}\text { Apple } \\
\text { PowerBook* }\end{array}$ & $\begin{array}{c}\text { Apple } \\
\text { Power Mac }\end{array}$ & $\begin{array}{l}\text { Compaq } \\
\text { Armada* }\end{array}$ & $\begin{array}{l}\text { Compaq } \\
\text { Presario }\end{array}$ & $\begin{array}{c}\text { Dell } \\
\text { Latitude* }\end{array}$ & $\begin{array}{c}\mathrm{HP} \\
\text { Omnibook* }^{*}\end{array}$ & $\begin{array}{c}\text { HP } \\
\text { Pavilion }\end{array}$ & $\begin{array}{l}\mathrm{IBM} \\
\mathrm{PC}\end{array}$ & $\begin{array}{c}\text { IBM } \\
\text { Thinkpad* }\end{array}$ \\
\hline \multicolumn{10}{|l|}{ price elasticities } \\
\hline PowerBook ${ }^{*}$ & -12.861 & 0.0692 & 0.0243 & 0.0287 & 0.0170 & 0.0219 & 0.0213 & 0.0182 & 0.0165 \\
\hline Power Mac & 0.0856 & -11.097 & 0.0202 & 0.0222 & 0.0196 & 0.0202 & 0.0248 & 0.0298 & 0.0364 \\
\hline Armada $7 x x x^{*}$ & 0.0150 & 0.0107 & -5.7066 & 0.0193 & 0.0606 & 0.0209 & 0.0203 & 0.0162 & 0.0426 \\
\hline Presario 2xxx & 0.0122 & 0.0272 & 0.0125 & -3.6032 & 0.0230 & 0.0272 & 0.0308 & 0.0348 & 0.0385 \\
\hline Latitude XPI* & 0.0263 & 0.0274 & 0.0357 & 0.0261 & -5.5701 & 0.0225 & 0.0217 & 0.0394 & 0.0453 \\
\hline Omnibook $4 \mathrm{xxx}^{*}$ & 0.0179 & 0.0147 & 0.0363 & 0.0298 & 0.0228 & -5.6501 & 0.0269 & 0.0222 & 0.0499 \\
\hline Pavilion 6xxx & 0.0118 & 0.0212 & 0.0153 & 0.0336 & 0.0167 & 0.0227 & -5.1178 & 0.0396 & 0.0359 \\
\hline$P C 3 x x x$ & 0.0137 & 0.0322 & 0.0137 & 0.0381 & 0.0153 & 0.0148 & 0.0325 & -3.2626 & 0.0215 \\
\hline Thinkpad 7xxx & 0.0330 & 0.0192 & 0.0376 & 0.0195 & 0.0304 & 0.0425 & 0.0297 & 0.0291 & -6.9745 \\
\hline \multicolumn{10}{|c|}{ advertising semi-elasticities } \\
\hline PowerBook ${ }^{*}$ & 0.0076 & -0.0057 & -0.0142 & -0.0110 & -0.0044 & -0.0139 & -0.0166 & -0.0072 & -0.0097 \\
\hline Power Mac & -0.0057 & 0.0215 & -0.0147 & -0.0273 & -0.0179 & -0.0136 & -0.0243 & -0.0263 & -0.0213 \\
\hline Armada $7 x x x^{*}$ & -0.0616 & -0.0564 & 0.0017 & -0.0057 & -0.0314 & -0.0625 & -0.0441 & -0.0684 & -0.0948 \\
\hline Presario $2 x x x$ & -0.0779 & -0.0827 & -0.0060 & 0.0120 & -0.0208 & -0.1092 & -0.1413 & -0.0825 & -0.0830 \\
\hline Latitude XPI* & -0.0233 & -0.0114 & -0.0278 & -0.0274 & 0.0230 & -0.0380 & -0.0239 & -0.0199 & -0.0438 \\
\hline Omnibook $4 \mathrm{xxx}^{*}$ & -0.0034 & -0.0042 & -0.0039 & -0.0043 & -0.0064 & 0.0054 & -0.0021 & -0.0030 & -0.0044 \\
\hline Pavilion 6xxx & -0.0036 & -0.0045 & -0.0038 & -0.0082 & -0.0051 & -0.0066 & 0.0101 & -0.0143 & -0.0054 \\
\hline$P C 3 x x x$ & -0.0076 & -0.0085 & -0.0082 & -0.0161 & -0.0182 & -0.0127 & -0.0194 & 0.0095 & -0.0029 \\
\hline Thinkpad 7xxx & -0.0107 & -0.0088 & -0.0168 & -0.0164 & -0.0185 & -0.0127 & -0.0196 & -0.0020 & 0.0089 \\
\hline
\end{tabular}

Table V: A Sample from 1998 of Estimated Price and Advertising Elasticities

\footnotetext{
${ }^{46}$ Elasticities are computed by multiplying the numerical derivative of estimated demand by price and dividing by actual sales.
} 
Estimated advertising demand elasticities indicate that, for some firms, advertising for one product has negative effects on other products sold by that firm but it is less negative than for some of the rival products. ${ }^{47}$ The lower panel presents a sample from 1998. Each semielasticity gives the percentage change in the market share of the row computer associated with a $\$ 1000$ increase in the (estimated) advertising of the column computer. For instance, a $\$ 1000$ increase in advertising for Apple Power Mac results in a decreased market share of around $0.1 \%$ for Compaq Presario but has very little effect on the market share for Apple PowerBook. In contrast, an increase in advertising for HP Omnibook has a large effect (relative to increase in own market share) on the market share for HP Pavilion.

To gain more insight into firms' advertising choices I use estimated demand to infer marginal costs and markups. Summary statistics are in Table I. The median markup charged by PC firms is $15 \%$ over marginal costs of production and $10 \%$ over per unit production and (estimated) advertising costs. As the first two rows show, the top firms have higher than average markups and advertising expenditures relative to the industry. Indeed the non-top firms' average median markup is much lower, $12 \%$, with an ad-to-sales ratio of about $2 \%$. The final column shows that, even after controlling for the fact that the top firms advertise more, they continue to earn higher than average markups. In 1998 the median industry markup was $19 \%$ over costs with the top firms earning a $22 \%$ markup. Overall industry and top firm markups were increasing over the period.

The bottom portion of the table gives details for top firms. Firms advertising choices are determined by their markup and their advertising elasticity of demand. IBM has one of the highest ad-to-sales ratios. IBM's demand is not more sensitive to advertising relative to other top firms however, IBM markups are higher than average. The results indicate that IBM is advertising more than the average non-top firm because they earn more per product than the average non-top firm. Compaq, on the other hand, has one of the highest markup margins (23\%) but still advertises less than average (although not less than the average nontop firm). As expected, Compaq's demand is less sensitive to advertising relative to other firms, which is the driving factor in their advertising decision. In addition, Gateway has the highest median price of the top firms but earns lower than average markups. The lower markups are due to higher costs, as reflected in a higher than average cost unobservable $(\omega)$, suggesting they are not as cost-effective in making their computers.

Effects of Limited Information The high estimated markups are explained in part by the fact that consumers know only some of the products for sale, due in part to the

\footnotetext{
${ }^{47}$ The model does not allow advertising for one product (or by one firm) to have positive spillovers to another product. Hence, the cross-product advertising effects (the off-diagonals in the lower panel of Table 5) are all negative. The diagonal elements report the increase in market share from own-advertising. For example, an increase of $\$ 1000$ for advertising on Dell Latitude results in an increased market share of $0.02 \%$.
} 
advertising decisions of firms. If all consumers had full information (the assumption made in the literature to date) the market would appear very different. Table VI compares the markups resulting from a model of limited information to those predicted by traditional models. I estimated a benchmark BLP model ${ }^{48}$ (the baseline model), which allows me to examine the additional markup firms earn as a result of limited consumer information. The estimates indicate median markups would be $5 \%$ under full information, one-third the magnitude of those under limited information.

The bottom rows present markup comparisons broken down by top firms with some representative products for each firm. The model of limited information suggests there is a larger markup gap between the top firms and the industry average, relative to the prediction under full information. Not surprisingly the firm with the largest percentage change in markups is IBM, the one that spends the most on advertising currently.

\begin{tabular}{|c|c|c|c|c|}
\hline & & \multicolumn{2}{|c|}{ Median Percentage Markup } & \multirow[b]{2}{*}{$\begin{array}{c}\text { Change } \\
\text { in Markups }\end{array}$} \\
\hline & & $\begin{array}{l}\text { Under Limited } \\
\text { Information }\end{array}$ & $\begin{array}{l}\text { Under Full } \\
\text { Information }\end{array}$ & \\
\hline Total industry & & $15 \%$ & $5 \%$ & $67 \%$ \\
\hline \multirow[t]{4}{*}{ Apple } & & & $2.5 \%$ & $84 \%$ \\
\hline & iMac & $22.1 \%$ & $3.1 \%$ & \\
\hline & Power Mac & $13.7 \%$ & $2.0 \%$ & \\
\hline & PowerBook* & $10.0 \%$ & $1.6 \%$ & \\
\hline \multirow[t]{5}{*}{ Compaq } & & & $7.0 \%$ & $69 \%$ \\
\hline & Armada $7 x x x^{*}$ & $41.4 \%$ & $3.5 \%$ & \\
\hline & Presario $2 x x x$ & $18.1 \%$ & $2.6 \%$ & \\
\hline & Presario $1 \mathrm{xxx}^{*}$ & $15.2 \%$ & $2.0 \%$ & \\
\hline & ProLinea & $23.3 \%$ & $7.0 \%$ & \\
\hline \multirow[t]{4}{*}{ Dell } & & & $1.8 \%$ & $82 \%$ \\
\hline & Latitude XPI* & $7.0 \%$ & $1.4 \%$ & \\
\hline & Dimension & $15.5 \%$ & $2.4 \%$ & \\
\hline & Inspiron & $9.4 \%$ & $1.6 \%$ & \\
\hline \multirow[t]{3}{*}{ Gateway } & & & $1.7 \%$ & $86 \%$ \\
\hline & Gateway Desk Series & $12.8 \%$ & $1.9 \%$ & \\
\hline & Gateway Portable Series & $8.1 \%$ & $1.5 \%$ & \\
\hline \multirow[t]{4}{*}{ HP } & & & $4.5 \%$ & $72 \%$ \\
\hline & OmniBook 4xxx* & $8.3 \%$ & $5.7 \%$ & \\
\hline & Pavilion 6xxx & $22.7 \%$ & $3.1 \%$ & \\
\hline & Vectra 5xx & $15.8 \%$ & $6.8 \%$ & \\
\hline \multirow[t]{4}{*}{ IBM } & & & $2.0 \%$ & $88 \%$ \\
\hline & Aptiva & $16.0 \%$ & $2.3 \%$ & \\
\hline & Thinkpad $7 x x x^{*}$ & $7.4 \%$ & $1.6 \%$ & \\
\hline & IBM PC 3xx & $26.1 \%$ & $2.1 \%$ & \\
\hline \multirow[t]{3}{*}{ Packard Bell } & & & $3.0 \%$ & $81 \%$ \\
\hline & NEC Versa* & $11.1 \%$ & $1.6 \%$ & \\
\hline & NEC Desk Series & $17.6 \%$ & $2.5 \%$ & \\
\hline
\end{tabular}

Table VI: Estimated Percentage Markups under Limited and Full Information

The extent to which a firm can exercise market power depends on the elasticity of its products demand curves. The greater the number of competitors or the larger the crosselasticity of demand with the products of other firms, the greater the elasticity of the firm's demand curve and the less its market power. A comparison of estimated product price elas-

\footnotetext{
${ }^{48} \mathrm{I}$ include the micro moments in the BLP model to obtain as precise estimates of the parameters of the taste distribution as possible (see Petrin, 2002). Parameter estimates are given in Goeree (2008).
} 
ticities for a sample of products is given in Table VII. The model of full information (bottom panel) presents an image of an industry that is quite competitive, and indicates markups are similar across products sold by the top firms. ${ }^{49}$ In addition, demand is very sensitive to price changes and cross-elasticities imply the products are somewhat substitutable. However, if we remove the full information assumption the industry looks very different. Firms have much more market power, as evidenced by the elasticities given along the diagonal in the top panel. Also cross-price elasticities indicate products are not as substitutable. This is intuitive, if consumers know of fewer products then products effectively face fewer competitors resulting in a less competitive industry.

\begin{tabular}{|c|c|c|c|c|c|c|c|c|c|c|c|c|}
\hline & $\begin{array}{c}\text { Apple } \\
\text { Performa }\end{array}$ & $\begin{array}{c}\text { Apple } \\
\text { PowerBook* }\end{array}$ & $\begin{array}{l}\text { Compaq } \\
\text { Contura* }\end{array}$ & $\begin{array}{l}\text { Compaq } \\
\text { Presario }\end{array}$ & $\begin{array}{c}\text { Dell } \\
\text { Latitude* }^{*}\end{array}$ & $\begin{array}{l}\text { Gateway } \\
\text { Desk }\end{array}$ & $\begin{array}{l}\text { Gateway } \\
\text { Portable* }\end{array}$ & $\begin{array}{c}\mathrm{HP} \\
\text { Pavilion }\end{array}$ & $\begin{array}{c}\mathrm{HP} \\
\text { Vectra }\end{array}$ & $\begin{array}{l}\text { IBM } \\
\text { PC }\end{array}$ & $\begin{array}{c}\text { IBM } \\
\text { Thinkpad* }^{*}\end{array}$ & $\begin{array}{c}\text { Pack Bell } \\
\text { Desk }\end{array}$ \\
\hline \multicolumn{13}{|l|}{ under limited information } \\
\hline Performa & -8.119 & 0.085 & 0.018 & 0.034 & 0.013 & 0.009 & 0.006 & & 0.021 & 0.019 & & 0.023 \\
\hline PowerBook Duo* & 0.061 & -11.568 & 0.024 & 0.023 & 0.009 & 0.007 & 0.018 & & 0.018 & 0.012 & & 0.028 \\
\hline Contura* & 0.014 & 0.010 & -8.929 & & 0.052 & 0.031 & 0.040 & & 0.012 & 0.013 & & 0.025 \\
\hline Presario $4 x x x$ & 0.011 & 0.011 & & -3.508 & & 0.009 & 0.009 & & 0.026 & 0.025 & 0.036 & 0.024 \\
\hline Latitude $^{*}$ & 0.027 & 0.009 & 0.025 & & -8.344 & 0.042 & 0.046 & & 0.011 & 0.009 & & 0.033 \\
\hline Gateway Desk Series & 0.015 & 0.008 & 0.034 & 0.105 & 0.008 & -3.955 & 0.008 & 0.030 & 0.014 & 0.012 & 0.006 & 0.027 \\
\hline Gateway Portable Series* & 0.029 & 0.015 & 0.055 & 0.037 & 0.013 & 0.015 & -6.757 & 0.018 & 0.022 & 0.020 & 0.079 & 0.015 \\
\hline Pavilion $4 x x x$ & & & & & & 0.133 & 0.026 & -5.173 & & & 0.045 & \\
\hline Vectra XU & 0.016 & 0.012 & 0.011 & 0.018 & 0.012 & 0.010 & 0.010 & & -5.534 & 0.026 & & 0.036 \\
\hline IBM PC 7xx & 0.013 & 0.007 & 0.029 & 0.019 & 0.007 & 0.008 & 0.037 & & 0.007 & -3.687 & & 0.086 \\
\hline Thinkpad $6 \mathrm{xx}^{*}$ & & & & 0.010 & & 0.026 & 0.080 & 0.024 & & & -5.209 & \\
\hline Packard Bell Desk Series & 0.008 & 0.005 & 0.003 & 0.018 & 0.007 & 0.006 & 0.004 & & 0.012 & 0.022 & & -3.317 \\
\hline \multicolumn{13}{|c|}{ under full information (blp benchmark) } \\
\hline Performa & -28.648 & 0.106 & 0.088 & 0.060 & 0.072 & 0.066 & 0.051 & & 0.097 & 0.090 & & 0.057 \\
\hline PowerBook Duo* & 0.089 & -31.654 & 0.047 & 0.099 & 0.060 & 0.058 & 0.046 & & 0.060 & 0.076 & & 0.060 \\
\hline Contura* & 0.065 & 0.080 & -31.721 & & 0.235 & 0.307 & 0.128 & & 0.050 & 0.038 & & 0.028 \\
\hline Presario $4 x x x$ & 0.025 & 0.013 & & -29.491 & & 0.038 & 0.099 & & 0.131 & 0.128 & 0.062 & 0.061 \\
\hline Latitude* $^{*}$ & 0.030 & 0.010 & 0.160 & & -29.547 & 0.195 & 0.175 & & 0.025 & 0.092 & & 0.076 \\
\hline Gateway Desk Series & 0.033 & 0.039 & 0.170 & 0.263 & 0.019 & -34.213 & 0.011 & 0.107 & 0.011 & 0.012 & 0.038 & 0.069 \\
\hline Gateway Portable Series* & 0.030 & 0.032 & 0.315 & 0.212 & 0.023 & 0.017 & -34.453 & 0.133 & 0.023 & 0.023 & 0.060 & 0.017 \\
\hline Pavilion $4 \mathrm{xxx}$ & & & & & & 0.135 & 0.019 & -35.362 & & & 0.090 & \\
\hline Vectra XU & 0.069 & 0.040 & 0.031 & 0.017 & 0.080 & 0.080 & 0.047 & & -39.009 & 0.011 & & 0.035 \\
\hline IBM PC 7xx & 0.149 & 0.138 & 0.180 & 0.236 & 0.060 & 0.081 & 0.078 & & 0.030 & -20.780 & & 0.209 \\
\hline Thinkpad $6 x^{*}$ & & & & 0.163 & & 0.080 & 0.056 & 0.069 & & & -39.809 & \\
\hline Packard Bell Desk Series & 0.028 & 0.031 & 0.185 & 0.213 & 0.050 & 0.048 & 0.045 & & 0.300 & 0.260 & & -26.327 \\
\hline
\end{tabular}

Table VII: Median Product Price Elasticities under Limited and Full Information

Results suggest (i) limited information about a product is a contributing factor to differences in purchase outcomes and (ii) information is distributed across households in a non-random way. Traditional full information models capture all differences in information through the additive unbounded iid term or the unobserved product characteristics term $(\xi)$, both of which are independent across households. Information heterogeneity indirectly captured by the iid error will be restricted such that each consumer/product pair has its own realization that is independent of consumer and product attributes (such as advertising)

\footnotetext{
${ }^{49}$ Bajari and Benkard (2004) estimate PC demand and find high implied demand elasticities (median own price elasticity -100) consistent with those I obtained from the BLP full information model. I discuss the Bajari and Benkard model in the next section and compare their model to the limited information model.
} 
and of all other consumer/product pairs. This doesn't permit correlation in information across consumers, nor does it permit informational advantages to depend on consumer and product observables. Alternatively, information heterogeneity can be indirectly captured via unobserved product characteristics. In the model of limited information, a product with little advertising is unlikely to be in many consumer's choice sets and will have a low market share. In the BLP model, a small market share could be explained by a low value for $\xi_{j}{ }^{50}$ Again, the unobserved term is independent of consumer attributes. Not explicitly allowing for informational asymmetries is particularly restrictive in rapidly changing markets where consumers are likely to have limited information, and hence where heterogeneity in the distribution of information across consumers and products explains (perhaps a significant) part of the variation in sales across products.

The results indicate that relying on an additive unbounded iid error term or unobserved product characteristics to explain differences in information across consumer-product pairs can generate inconsistent estimates of product specific demand curves that are biased towards being too elastic. Consider as an example a market that consists of three products each produced by a different firm. These products have identical characteristics, but the firms are each monopolies due to limited consumer information. That is, there are three groups of consumers where each group knows only one product. In this world, each of the three firms would earn monopoly markups. Let's consider how the full and limited information models would address the data generated from such a world.

First, assume the consumers are identical. In the data we would observe identical individuals purchasing different products with identical observed characteristics. The model would need to make the products differ somehow to match the data. Traditional models would rely on the iid term to explain the observed purchase patterns. (The model couldn't use different $\xi$ to match the data since all consumers would buy the product with the highest $\xi$.) Would this result change if consumers were heterogenous, that is could the model explain different purchases through different consumer tastes? No, because observed product characteristics are the same (i.e. a consumer with a large taste for CPU speed has to choose among three products with identical CPU speed, that are also identical in every other observable respect). There are two points here (i) the iid terms would allow the model to match the purchase patterns but would use random consumer-product variation to do so (ii) the estimated elasticities would be more elastic than the true elasticities. Estimated markups would be much lower than true markups.

The limited information framework could explain differences in choices among otherwise identical products through differences in consumer information across products. First, in the case of no advertising, the model permits information heterogeneity due to differences in con-

\footnotetext{
${ }^{50} \mathrm{I}$ thank an anonymous referee for this point.
} 
sumer attributes. Second, household information heterogeneity could arise if firms advertise products using different media where certain media are more effective at informing certain types of consumers. The limited information model allows consumers to be non-randomly differentially informed, which may explain differences in purchase patterns observed in the data. There are two points here (i) the limited information model matches purchase patterns using non-random information heterogeneity across consumers and products (relying less on the iid term) (ii) the estimated elasticities would be more inelastic than the traditional elasticities. In this example, the markups estimated from the limited information model would be higher relative to those obtained under traditional models.

Consider another example. The market again consists of three products with identical observed characteristics, but product one has a low market share relative to the others. Again, for the sake of illustration, assume product one is a high quality product where the unequal distribution of market shares is due to limited information: few consumers know product one. The BLP model can match the data through one of two ways (i) through the iid error term (ii) through unobserved product characteristics $(\xi)$. Since mean utility is chosen to match market shares, the model will force product one's mean utility to be lower through a low value of $\xi$. This has the implication that consumers are more sensitive to price changes in product one, ceteris paribus, since their mean utility is lower. However, in truth product one is a high quality product, and hence should have a high $\xi$ value. The limited information model would allow for the following: few consumers are informed about the existence of product one (perhaps due to low advertising for the product), implying it has a low market share. High quality implies a high value of $\xi$ resulting in higher utility for consumers who know the product, ceteris paribus, resulting in more inelastic demand among fewer consumers. The limited information model would predict that product one would have higher markups than those predicted by traditional models.

This is best illustrated by examining the differences in the value of the unobserved product characteristics terms when the parameters are estimated via BLP full information versus limited information models. Apple's PowerBook G3 was introduced in November 1997 and was designed to use a high speed "backside" cache which could interact with the processor at much faster speeds than a standard L2 cache (which was restricted by the motherboard speed). At the time, the PowerBook G3 was considered the fastest notebook in the world. It received very favorable reviews for its speed, weight, size, design, and overall performance. ${ }^{51}$ The PowerBook G3 had a very small share of the market, both because Apple's market share was low during this period (around 6\%) and also because the PowerBook G3 was only on the market for 5 months. To match the low market shares, the BLP model generates a low value of $\xi$ (relative to other products in the quarter). In contrast, the limited information

\footnotetext{
${ }^{51}$ apple-history.com;pcworld.com/article/id,11954/article.html; epinions.com; consumerreports.com
} 
model generates a low average value for $\phi$, but a significantly higher $\xi$ value than in the BLP model. The anecdotal evidence seems to support the limited information results: there are few consumers who know the PowerBook G3, but among the informed subset mean utility is increased from buying it, ceteris paribus. This suggests Apple could earn a high markup on the PowerBook G3 from the subset of consumers who know it. Indeed, the estimated limited information markups for PowerBook G3 are on the order of 11\%, while the BLP estimates suggest this is a product with low markups (around 1\%). The results suggest that traditional models, which rule out non-random informational asymmetries across households and products a priori, yield inconsistent estimates for product specific elasticities that are biased towards being too elastic. ${ }^{52}$

\section{Sensitivity Analysis}

I examine the robustness of the limited information model by conducting goodness-of-fit tests. First, I tested whether all the moments were satisfied. The objective function is a Wald statistic distributed chi-squared with degrees of freedom equal to the number of moment restrictions less the number of parameters. This test is conditional on all assumptions of the model and tests the overidentifying moment restrictions together with all functional form and distributional assumptions. The test is stringent and generally rejects for large samples. It is not surprising then, given the large sample size and stylized nature of the model, that the model is rejected by the data.

Second, I conducted goodness-of-fit tests focused on various aspects of the model. I partitioned the region in which the response variables (and in some cases covariates) lie into disjoint cells. I calculated the quadratic form based on the difference between the observed number of outcomes in each cell and the expected number (given the observed covariates). If the model is correct, the normalized quadratic form converges in distribution to a chi-square random variable as the sample size increases. ${ }^{53}$

Formal tests were not able to reject the null that predicted values for market shares are the same as the observed values. ${ }^{54}$ I also constructed test statistics based on the average value of shares that fall into specified cells. Again, the test statistic is below the $10 \%$ level of significance critical value: the null hypothesis is not rejected. Controlling for product attributes, the model does a good job of predicting average market shares across

\footnotetext{
${ }^{52}$ See Goeree $(2002,2008)$ for details concerning why full information and limited information models will (most likely) result in different estimates for price elasticities of demand.

${ }^{53}$ These tests are based on those presented in Andrews(1988). The predetermined number of cells are centered at the mean of the response variable with a width proportional to its standard deviation.

${ }^{54}$ The test statistic is chi-squared with 7 degrees of freedom. The realized value (4.7) is below the $10 \%$ level critical value (12). The model fits well, but misses more among lower market share products.
} 
cells. However the model tends to miss more among non-Pentiums.

Third, I compared the limited information model (hereafter LIM) to three alternatives. The first is the BLP model (with micro moments). The second is a full information model where advertising affects the utility function directly. I refer to this model as the uninformative model (hereafter UN). The third is a modification of the BLP model proposed by Bajari and Benkard (2005, hereafter BB). They estimate PC demand and find high estimated own-price elasticities. They (independently) attribute their unrealistically high estimates to the full information assumption. They estimate a modified BLP model limited to those products with large market shares. The intuition being consumers are more likely to know these products since it is easier to obtain information on them. ${ }^{55}$

I would prefer to be able to test the relative fit of the models parametrically. Unfortunately a formal test of non-nested hypotheses (Vuong, 1989) would require additional assumptions on the distribution of the errors. While the data suggest no natural assumptions for the error distributions I present analysis that highlights the strengths and weaknesses of the fit of LIM relative to other models. For instance, both LIM and UN predict a threshold level of average group ad expenditures (above which products will be advertised in groups and below which they will be advertised individually). We should never observe group (product specific) expenditures below (above) this level. The LIM and UN models predict different threshold levels. These predictions are presented in the second panel of Table VIII. The LIM model misses about $3 \%$ of the time, while UN misses more than twice as much, $8 \%$. Most of the misses for both models are among Apple products $(2.4 \%$ for LIM and $8 \%$ for UN), while both models' predictions match the data for HP and Packard Bell. In addition, both models miss more among TV advertisements (1.5\% for LIM and $5.5 \%$ for UN). The fact that UN fits worse in this dimension is not surprising since UN predicts a higher threshold level (so we expect to observe a larger percentage of group expenditures below the predicted threshold). It is surprising that LIM does no worse than UN regarding the proportion of product specific expenditures above the predicted threshold. Both models miss less than 1\% on average, with all the misses coming among Apple and Compaq products. This anecdotal evidence suggests, at the very least, that the LIM model fits no worse than the UN model.

Another dimension along which the models can be compared regards the role of unobserved product attributes. In all models mean utility is chosen such that predicted shares match observed shares. While there is no explicit role for advertising in the BLP model or $\mathrm{BB}$ modification, one can interpret the unobserved product heterogeneity terms $\left(\xi_{j}\right)$ as containing product advertising. ${ }^{56}$ Using the parameter estimates from the respective models, I

\footnotetext{
${ }^{55}$ The parameter estimates for the alternative models can be found in Goeree (2008).

${ }^{56}$ In the LIM model, a product with little advertising is unlikely to be in many consumer's choice sets and will have a low market share. In the BLP and BB models, a small market share would be explained by a
} 
restricted $\xi_{j}$ to zero and recalculated the predicted market shares. These "pseudo"predicted shares are presented in the first panel of Table VIII. These provide insight into the importance of unobserved product attributes in each model as well as indicate how well the model fits market shares based solely on observables and the form of the model.

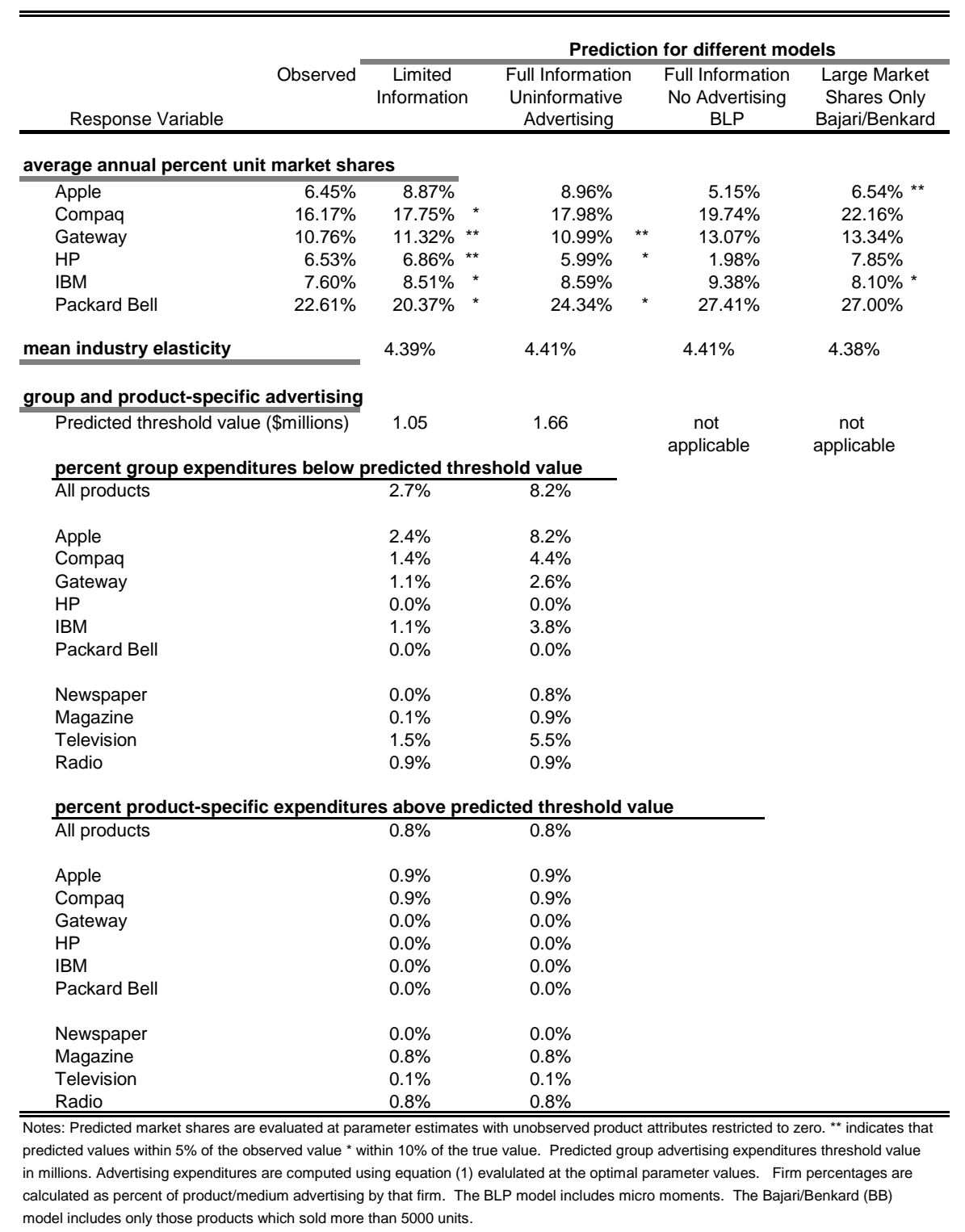

\section{Table VIII: Goodness of Fit}

The BLP model's predicted pseudo shares do not come within $10 \%$ of the observed market shares for any of the top firms (second to last column). The BB modification (last column) fits the market shares of the top firms better than the BLP model, the Apple shares are within $5 \%$ of the observed shares and IBM within 10\%. This is not surprising since BB low value for $\xi_{j}$. 
restricts estimation to the larger firms. Both $\mathrm{BLP}$ and $\mathrm{BB}$ provide a worse fit than the models in which advertising plays an explicit role. Again, this is not a surprise as the $\xi_{j}$ play a larger role in the BLP and BB model relative to the advertising models. The LIM model fits the market shares better than the UN model. For Gateway and HP, the pseudo market shares are within 5\% of observed shares and for Compaq, IBM, and Packard Bell, the pseudo shares are within 10\%. The UN model comes within $5 \%$ of the observed market shares for Gateway and within 10\% for HP and Packard Bell. Neither model predicts Apple market shares within 10\%. This is perhaps not so surprising given that the firm for which the advertising predictions miss the most is Apple. These results suggest the model of limited information does a good job of predicting advertising and market shares in the PC industry, relative to models in which consumers are assumed to be aware of all products.

While the alternative models present different pictures of product elasticities, they are consistent in their predictions of industry elasticities. For all models, I simulate a $1 \%$ increase in the price of all (inside) goods and calculate the percentage change in total home market share. Mean industry elasticities are given in the second panel. Industry demand is more inelastic, an intuitive result given the relative scarcity of products which are substitutable for PCs (particularly over this time frame).

Due to the difficulty in obtaining ad data for some industries, a comparison of BLP and $\mathrm{BB}$ may be useful. If LIM is believed to be the correct model, then the BB modification may be preferred in that it generates estimates of product demand curves that are less elastic (relative to BLP) and closer in magnitude to those of LIM. ${ }^{57}$ However, the $\xi_{j}$ still play a large role in BB, namely only for Apple is the reliance on the $\xi_{j}$ small enough to provide an adequate fit of market shares based on observables. To the extent that the role (or number of) smaller firms is an important dimension of industry competition, the BB modification will not be preferred to other models of full information.

Recall, that LIM restricts attention to the top ten firms plus five other small firms. This sample selection could effect estimated margins in two ways. First the smaller products not included in the sample are likely to have higher own-price elasticities and hence lower markups (relative to similar included products). Estimated markups for the included products will be higher the more smaller firms (or less-advertising intensive firms) are excluded. This effect would be in largest for the BB modification which limits the sample to large firms. Indeed BB found evidence of much higher markups (less elastic demand curves). This effect is less pronounced for LIM in that five of the firms are small. The other effect of limiting the sample has to do with the impact on the "outside" good. The fewer products are included among the "inside" goods (the larger is the outside good) the lower will be

\footnotetext{
${ }^{57} \mathrm{BB}$ find estimated product specific demand elasticities ranging from -4 to -72 with a median elasticity of -11 for their modified model.
} 
estimated markups for the inside goods. Under full information when a product is added to the sample that product is a competitor with every other product. The overall impact on markups will depend upon the substitution patterns among the inside goods and the size of the outside good. However when a product is added to the LIM model it may not be a competitor with every other product (some consumers may not know it exists). Hence, LIM markups will not be as sensitive to adding new firms to the included sample as will models of full information.

Modeling advertising as affecting a consumer's choice set requires significant computation time since the choice sets must be simulated. To test if the benefits of simulating choice sets are worth the costs of increased computation time, I performed a monte-carlo experiment. Consider a market consisting of two products and one outside good. Denote the probability consumers are aware of a product by $\phi_{j}$. The limited information market share is

$$
s_{1}=\phi_{1}\left(1-\phi_{2}\right) \frac{D_{1}}{1+D_{1}}+\phi_{1} \phi_{2} \frac{D_{1}}{1+D_{1}+D_{2}}
$$

where $D_{j}$ represents $\exp \left(\delta_{j}\right)$, the mean utility from product $j$, analogously for product 2 . A version of the market share which would not require simulating choice sets is

$$
s_{1}^{*}=\frac{\phi_{1} D_{1}}{1+\phi_{1} D_{1}}+\frac{\phi_{1} D_{1}}{1+\phi_{1} D_{1}+\phi_{2} D_{2}} .
$$

I calculated the values of $s_{j}$ and $s_{j}^{*}$ for different values of $\phi$ and $D$. The resulting value of $s_{j}^{*}$ was within $5 \%$ of the value of $s_{j}$ only $2 \%$ of the time. Notice also that the specification for $s_{j}^{*}$ is not separately identifiable from a model in which advertising enters the utility function directly (or a model in which advertising is included in $\xi_{j}$ ). This obtains by defining $\phi^{*}=$ $\ln (\phi)$ and $D=\exp \left(\delta+\phi^{*}\right)$. These results suggest that the more computationally demanding LIM model cannot be replaced easily with a simplified version. Secondly, advertising which influences consumers' choice sets has very different effects from that which shifts demand directly through utility. That is, the standard BLP model and models in which advertising are one of the observed product attributes are not observationally equivalent to the model presented in this research. ${ }^{58}$

\section{Conclusions}

In markets characterized by rapid change, such as the PC industry, it is probable that consumers know only a subset of all available products. Models estimated under the assumption

\footnotetext{
${ }^{58} \mathrm{~A}$ model that includes both effects of advertising, through the choice set and directly in utility, is, theoretically, separately identifiable. However, in practice, one would like identification to be driven by variation in the data. See Ackerberg $(2001,2003)$ who uses micro data to estimate a model which allows for informative and uninformative effects of advertising.
} 
of full information present an image of the PC industry that is quite competitive. For example, a BLP full information model yields modest estimated median markups of $5 \%$. When we remove the full information assumption the industry looks very different. Indeed, estimated cross-price elasticities indicate products are not as substitutable as full information estimates suggest. I estimate a model of limited consumer information, where firms provide information through advertising. I find estimated median markups in the PC industry are high: $19 \%$ over production costs in 1998, where the top firms engage in higher than average advertising and earn higher than average markups. The results suggest firms have significant market power due in part to limited consumer information. The differences in estimated price elasticities (and implied markups) across the approaches reflects the inconsistency in the full information model which does not allow consumers to be differentiated in terms of information. I extend the BLP framework to permit systematic (non-random) differences in information based on consumer observables. I try to capture potential correlation in information across consumers using differences in information exposure across consumer types based on media exposure choices. The model allows for the possibility that the imperfect substitutability between different brands of consumer products is due to consumers' having limited information about product offerings as well as to consumer-idiosyncratic brand preferences. I show how to use additional data on media exposure to improve estimated price elasticities, á la BLP, in the absence of micro ad data.

The results suggest that (i) allowing for heterogeneity in consumers' choice sets yields more realistic estimates of substitution patterns between goods, (ii) assuming full information may result in incorrect conclusions regarding the intensity of industry competition, and (iii) firms benefit from limited consumer information. I find that exposure to advertising significantly impacts consumers' information sets, but that advertising has very different informative effects across individuals and across media. The estimates suggest that some firms are more effective at informing consumers through advertising. For some firms advertising one product can have a negative effect on the market share of other products sold by that firm, but the effect is less negative than it is for most of the rivals' products. There are economies of scope in group advertising and some firms find it worthwhile to engage in group advertising for some product lines to capitalize on the increasing returns.

Considering the implications of limited information is particularly important when addressing policy issues. In the PC industry, models estimated under the assumption that consumers are aware of all products generate estimates of product-specific demand curves that are biased towards being too elastic. The results of this paper suggest that antitrust authorities may reach different conclusions regarding the welfare implications of mergers depending on their assumptions regarding consumer information. ${ }^{59}$

${ }^{59}$ See Goeree (2002, 2003). 


\section{Appendix A. Approximations to the Optimal Instruments}

To motivate the instruments discussed in section 4, it easiest to first consider a simpler context. The following text borrows heavily from the Appendix to BLP (1999). The full information linear model has an estimating equation of

$$
\ln \left(s_{j}\right)-\ln \left(s_{0}\right) \equiv \delta_{j}=x_{j} \beta-\alpha p_{j}+\xi_{j} .
$$

The optimal instruments are $E(x \mid z)$ and $E(p \mid z)$ assuming $\xi$ is iid. Given that $x$ is an element of $z, E(x \mid z)=x$. If the demand and cost unobservable have some known density which is independent of $z$ then

$$
E(p \mid z)=\int p(x, w, \theta, \xi, \omega) f(\xi, \omega) d \xi d \omega
$$

where $p(x, w, \theta, \xi, \omega)$ is the equilibrium pricing function, which has as arguments the observed $(x, w)$ and unobserved $(\xi, \omega)$. BLP suggest using a series of basis functions to form a semiparametric approximation to $E(p \mid z)$. BLP (1999) suggest an approach that makes greater use of the functional form of equilibrium prices as implied by the model. I use this approach and outline it below.

In the case of (A.1) they propose to replace the expected equilibrium price in (A.2) with the equilibrium price at the expected value of the unobservables (ie at $\xi=\omega=0$ ). The instrument for price is then

$$
\widehat{p}=\left.p(x, w, \widehat{\theta}, \xi, \omega)\right|_{\xi=\omega=0}
$$

for some initial estimate of $\widehat{\theta}$. Note if the $x$ characteristics imply, given $\widehat{\theta}$ and $\xi=0$, that product $j$ has close rivals then the predicted markup for product $j$ will be low and its predicted price will be close to predicted marginal cost, $w_{j}^{\prime} \widehat{\eta}$. Otherwise, if a good is predicted to have no close rivals the instrument associated with price may be well above predicted marginal cost. As BLP note, rivals' characteristics have an effect on the calculated instrument that is motivated by the model.

It is trivial to extend the simple model in (A.1) to one in which advertising enters linearly. The corresponding instrument for advertising would be

$$
\widehat{a}=\left.a\left(x, w^{a d}, \widehat{\theta}, \xi, \omega\right)\right|_{\xi=\omega=0}
$$

for some initial estimate of $\widehat{\theta}$. Firms advertising choices depend on their markup, their advertising elasticities of demand, and the cost of advertising in different media. If the product has low predicted markups (due to many close rivals) then marginal revenue from advertising will be lower, ceteris paribus, and our predicted advertising (in each media) will be lower as well. ${ }^{60}$ Otherwise, if a good is predicted to have no close rivals predicted advertising will be higher, ceteris paribus. Note also that the level of predicted advertising for $j$ in media $m$ depends on the predicted marginal cost of advertising in that media $\left(w_{j m}^{a d} \widehat{\psi}\right)$.

The estimator will be biased since the price (advertising) evaluated at the expected values are not the expected value of price (advertising). However, the approximation is consistent since it is a function of exogenous data and is constructed to be highly correlated with the relevant functions of prices (advertising). Applying the method to the non-linear limited information model is more complex, but the instruments are still functions of the same exogenous data and are constructed in a way that makes use of the functional form of equilibrium prices and advertising implied by the model.

\footnotetext{
${ }^{60}$ Indeed if products are identical, Bertrand competitors will find it optimal not to advertise.
} 
The efficient set of instruments when we have only moment restrictions is

$$
E\left[\frac{\partial \xi_{j}\left(\Theta_{0}\right)}{\partial \theta}, \frac{\partial \omega_{j}\left(\Theta_{0}\right)}{\partial \theta} \mid z\right] T\left(z_{j}\right)
$$

where $T\left(z_{j}\right)$ is the matrix that normalizes the error matrix (Chamberlin, 1987). ${ }^{61}$ BLP (1999) propose to replace the expectation with the appropriate derivatives evaluated at the expectation of the unobservables. Below are the steps I take to construct such derivatives for the limited information model:

(i) Construct initial instruments for prices $\left(\widehat{p}_{\text {initial }}\right)$ and advertising. ${ }^{62}$

(ii) Use the initial instruments to obtain an initial estimate of the parameters, $\widehat{\Theta}$.

(iii) Construct estimates of $\delta, m c$, and $m c^{a d}$. I used $\widehat{\delta}=x \widehat{\beta}, \ln (\widehat{m c})=w \widehat{\eta}$, and $\ln \left(\widehat{m c}^{a d}\right)=$ $w^{a d} \widehat{\psi}$.

(iv) Solve the first-order conditions for equilibrium advertising, $\widehat{a}$, as a function of $(\widehat{\Theta}, \widehat{\delta}, \widehat{m c}$, $\left.\widehat{m c}^{a d}, \widehat{p}_{\text {initial }}, x\right)$.

(v) Solve the first-order conditions of the model for equilibrium prices, $\widehat{p}$, as a function of $(\widehat{\Theta}, \widehat{\delta}, \widehat{m c}, \widehat{a}, x)$.

(vi) These imply a value for predicted market shares, $\widehat{s}$, which is a function of $(\widehat{\Theta}, \widehat{p}, \widehat{\delta}, \widehat{a}, x)$.

(vii) This gives the unobservables evaluated at the exogenous predictions: $\widehat{\xi}(\theta)=\widehat{\xi}(\widehat{p}, \widehat{a}, \widehat{s}, \widehat{\delta}, x, \theta)$ and $\widehat{\omega}(\theta)=\widehat{\omega}(\widehat{p}, \widehat{a}, \widehat{s}, \widehat{\delta}, \widehat{m c}, x, \theta)$. Calculate the required disturbance-parameter pair derivatives.

(viii) Repeat steps (iv)-(vii) where each time the new $\widehat{p}_{\text {initial }}$ is replaced by the $\widehat{p}$ found from the previous round.

(ix) Form approximations to the optimal instruments by taking the average of the exogenous derivatives found in step (vii).

\section{Appendix B. Media Exposure Exogeneity Tests}

I use data from the Simmons survey to test whether media exposure is endogenous to the purchase decision. These include information on whether the individual purchased a PC, demographic characteristics, and media exposure information. For ease of exposition, assume there is only one advertising medium. ${ }^{63}$ The value to $i$ of purchasing a $\mathrm{PC}$ is

$$
y_{i}^{*}=z_{1 i} \delta_{1}+\alpha E_{i m}+u_{i}
$$

which depends upon exogenous control variables, $z_{1 i}$, (potentially endogenous) media exposure variables $E_{i m}$, and parameters. The explanatory variables included in $z_{1 i}$ are measures

\footnotetext{
${ }^{61}$ In the linear model of (A.1) $\partial \xi_{j} / \partial(\beta, \alpha)=\left(x_{j}, p_{j}\right)$.

${ }^{62}$ I constructed a distance variable based on observables and used kernel estimates for prices and advertising as the initial instruments.

${ }^{63} \mathrm{It}$ is straightforward to extend the framework to allow for multiple endogenous variables.
} 
of age, education, marital status, household size, gender, race, and income. We observe a purchase $y_{i}=1\left(y_{i}^{*}>0\right)$.

The amount of exposure of $i$ to medium $m$ is

$$
E_{i m}^{*}=z_{1 i} \delta_{21}+z_{2 m} \delta_{22}+\varepsilon_{i m}=Z_{i m} \delta_{2}+\varepsilon_{i m} .
$$

The instruments, $z_{2 m}$, are variables that impact exposure to medium $m$ but do not affect the probability you buy a PC, conditional on exposure. These consist of the price of media access and are discussed below. I assume $\left(u_{i}, \varepsilon_{i m}\right)$ has a mean zero, bivariate normal distribution and is independent of $Z_{i m}$.

Simmons reports the quintile into which the consumer falls with regard to media exposure. Defining quintile one as the highest, $i$ belongs to the $q$ th quintile in medium $m$ if $c_{q m}<E_{i m}^{*}<c_{(q-1) m}$ where $c$ are cutoff values. I construct a binary variable equal to one if $i$ falls in one of the top two quintiles for medium $m .{ }^{64}$

Smith and Blundell (1986) and Rivers and Vuong (1988) develop a two-step test for the exogeneity of regressors in limited dependent variable models. Wooldridge (2002) shows that the test of exogeneity is valid without assuming normality or homoskedasticity of $\varepsilon_{i m}$ and can be applied broadly even if the endogenous regressor is a binary variable. I present the results from the two-step regressions and the exogeneity tests after discussing the instruments.

If individuals consult a magazine or newspaper prior to purchase, they may buy a single copy off the newstand. Hence, I gathered data on two measures of the price of access: single-copy price and the per-issue price based on an annual subscription. I collected access prices for over 140 magazines and over 100 newspapers in 1996 and 1997 from the Audit Bureau of Circulations. Newspaper prices are averaged within 12 geographic regions.

Television viewers who fall into the highest quintiles may have access to more channels than those provided by a basic cable subscription (i.e. expanded basic cable). I use two measures of the cost of access to cable: the monthly fee for basic cable and the monthly fee for expanded basic cable. In some geographic regions, consumers can purchase pay service stations at an additional fee (HBO, Showtime, etc). Since these pay service stations are typically commercial free, I don't include the additional monthly fee associated with pay service access. The cable price data are from the Television and Cable Factbook, 1996 and 1997. I gathered data on access prices for over 250 cable carriers in 12 geographic regions. Cable prices are averaged within geographic region.

Table B1 presents the results with single-copy magazine, single-copy newspaper, and the monthly fee for expanded basic cable as instruments for exposure to magazines, newspapers, and television, respectively. The results indicate high income married individuals are more likely to be in the top quintiles of newspaper and magazine readership, where readership of newspapers is increasing in household size. Not surprisingly, low income individuals are more likely to be in the top $40 \%$ of exposure to television, where these individuals are less likely to be in the top quintiles for newspaper or magazine readership.

The first part of the lower panel reports the $\chi^{2}$ test-statistic of the restriction that access prices have no impact on exposure falling in the top $40 \%$ for each media. ${ }^{65}$ As indicated by the last row, the instruments have power in all specifications. The second panel presents the results of a Wald test of the null hypothesis that exposure is exogenous for each media. In all specifications, the test statistic is not significant, indicating that I cannot reject the null that exposure to newspapers, magazines, and cable television is exogenous to the PC purchase decision. I conducted the exogeneity tests for exposure to each quintile separately and for the alternative access prices mentioned above. The results do not change.

\footnotetext{
${ }^{64}$ Probit results suggest falling into the lower three quintiles does not significantly impact PC purchase probabilities, conditional on observed covariates. However, I conduct the same tests for exposure to each quintile separately and the results do not change.

${ }^{65}$ The price coefficient for newspaper is positive. This could reflect the higher quality (and prices) of newspapers read by individuals in the top quintiles.
} 


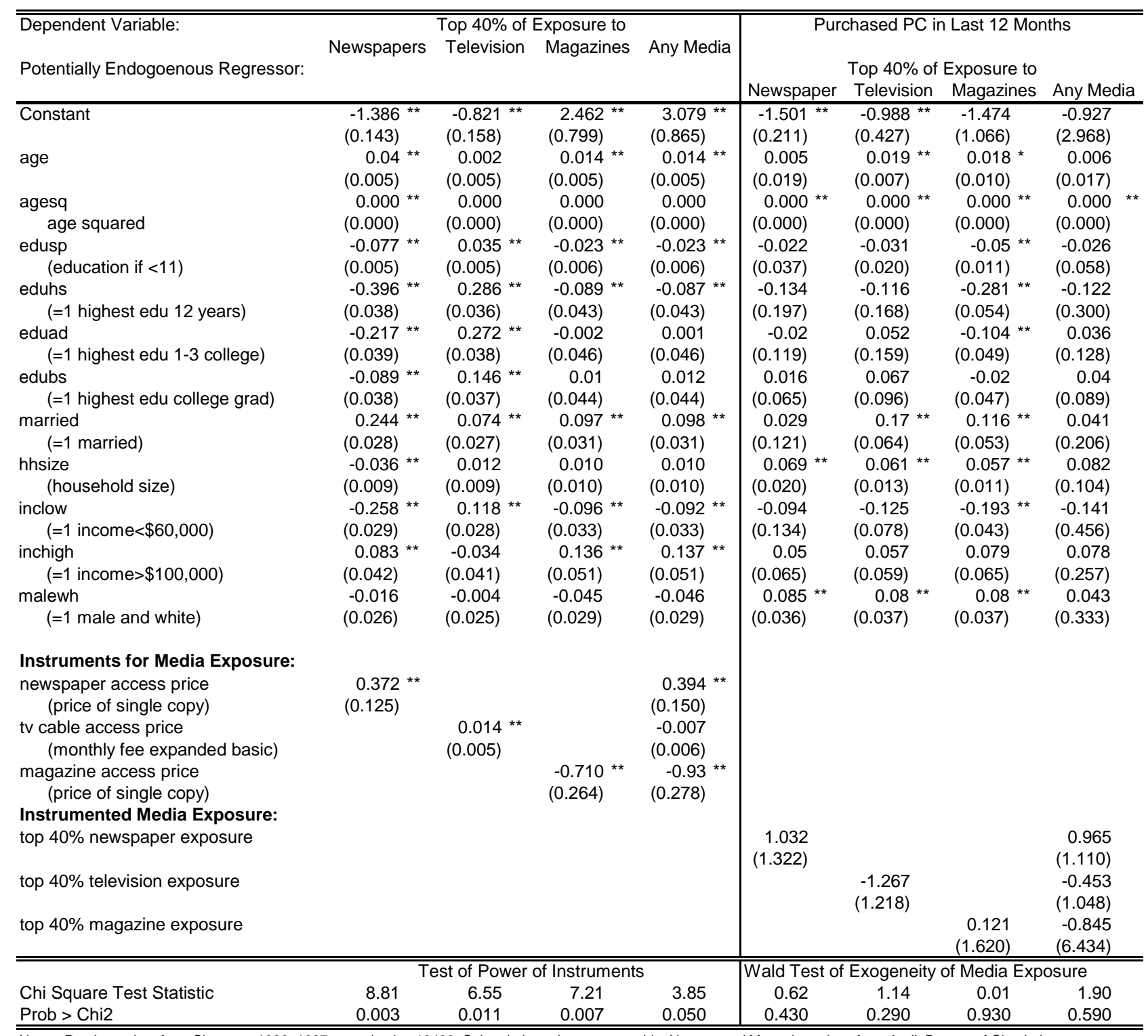

Notes: Purchase data from Simmons 1996, 1997; sample size 13400. Subscription prices are monthly; Newspaper/ Magazine prices from Audit Bureau of Circulation;

Cable prices from Television and Cable Factbook. Any media includes newspaper, magazine and tv. Standard errors in parenthesis. ${ }^{* *}$ significant at $5 \%$ and ${ }^{*}$ at $10 \%$.

Table B1: Two-Step Probit Exogeneity Tests

\section{Appendix C. Simulation Details}

A general outline for simulation follows, I omit the time subscript for clarity. First prepare random draws, which, once drawn, do not change throughout estimation.

1. In the case of the macro moments,

(a) Draw $i=1, \ldots, n s$ consumers from the joint distribution of characteristics and income given by the CPS, $G(D, y)$, and corresponding draws from multivariate normal distribution of unobservable consumer characteristics, $G(\nu)$, one for each product characteristic, call these $\nu_{i k}$ (where I drew a sample of 3000 for each year, $n s=9000)$ 
(b) Draw log normal variables one for each medium combination, call these $\kappa_{i m}$ (where $m=1, \ldots, 4)$.

(c) Draw uniform random variables one for each product-individual pair, call these $u_{i j}$

2. For the micro moments

(a) For each Simmons consumer $i=1, \ldots$, ncons draw $R$ times from multivariate normal distribution of unobservable consumer characteristics, $G(\nu)$, one for each product characteristic, call these $\nu_{i k r}($ where ncons $=13400)$.

(b) Draw $R$ uniform random variables for each product-individual combination, call these $u_{i j r}$.

(c) Draw $R$ log normal variables one for each medium-individual combination, call these $\kappa_{i m r}$.

3. Choose an initial value of the parameters $\theta_{0}$

4. For the macro-moments, do for $i=1, \ldots, n s$

(a) Calculate $\phi_{i j}(\theta)$ for each product $j=1, \ldots, J$ for each period

$$
\begin{gathered}
\phi_{i j}(\theta)=\frac{\exp \left(\tau_{i j}\right)}{1+\exp \left(\tau_{i j}\right)} \\
\tau_{i j}=\sum_{d} \widetilde{D_{i d}} \widetilde{\lambda}+\vartheta x_{j}^{a g e}+\sum_{m} \varphi_{m} a_{j m}+\sum_{m} \rho_{m} a_{j m}^{2}+\Psi_{f} \sum_{m} a_{j m}+\varsigma \sum_{m} \sum_{d} \Upsilon_{m d} D_{i d}^{s} a_{j m}+\sum_{m} a_{j m} \kappa_{i m}
\end{gathered}
$$

(b) Given $\phi_{i j}(\theta)$ and $u_{i j}$ construct a $J$ dimensional Bernoulli vector, $b_{i}(\theta)$. This defines the choice set $\mathcal{S}^{\prime}$, where the $j$ th element is determined according to

$$
b_{i j}= \begin{cases}1 & \text { if } \phi_{i j}(\theta)>u_{i j} \\ 0 & \text { if } \phi_{i j}(\theta) \leq u_{i j}\end{cases}
$$

Define $b_{i}^{0}$ to be the Bernoulli vector generated from the initial choice of parameters, $\theta_{0}$.

(c) Calculate

$$
P_{i j}(\theta)=\frac{\exp \left\{\delta_{j}+\mu_{i j}\right\}}{y_{i}^{\alpha}+\Sigma_{k: b_{i, k}^{0}=1} \exp \left\{\delta_{k}+\mu_{i k}\right\}}
$$

where $\mu_{i j}$ is value of $\alpha \ln \left(y_{i}-p_{j}\right)+\sum_{k} x_{j k}\left(\sigma_{k} \nu_{i k}+\sum_{d} \Omega_{k d} D_{i d}\right)$ given the $i$ th draw and $\theta$.

(d) Calculate

$$
s_{i j}(\theta)=\Pi_{l \in \mathcal{S}} \phi_{i l} \Pi_{k \notin \mathcal{S}}\left(1-\phi_{i k}\right) \frac{P_{i j}(\theta)}{\phi_{i}^{0}\left(\theta_{0}\right)}
$$

where $\phi_{i}^{0}\left(\theta_{0}\right)$ is the value of $\Pi_{l \in \mathcal{S}_{0}} \phi_{i l} \Pi_{k \notin \mathcal{S}_{0}}\left(1-\phi_{i k}\right)$ using the initial value of the parameters and the initial choice set. During estimation the parameter values will be updated so the simulated product over the $\phi_{i j}$ will differ from the initial $\phi_{i}^{0}\left(\theta_{0}\right)$ in all but the first simulation. 
5. Calculate the simulator for the market share

$$
\widehat{s_{j}}=\frac{1}{n s} \sum_{i} s_{i j}
$$

6. For the micro-moments: For each consumer, $i=1, \ldots$, ncons, calculate $\overline{\tau_{i j}}$

$$
\overline{\tau_{i j}}=\sum_{d} \widetilde{D_{i d}^{s}} \widetilde{\lambda}_{d}+\vartheta x_{j}^{a g e}+\sum_{m} \varphi_{m} a_{j m}+\sum_{m} \rho_{m} a_{j m}^{2}+\Psi_{f} \sum_{m} a_{j m}+\varsigma \sum_{m} \sum_{d} \Upsilon_{m d} D_{i d}^{s} a_{j m}
$$

do for $r=1, \ldots, R$ draws

(a) Calculate $\phi_{i j r}(\theta)$

$$
\begin{gathered}
\phi_{i j r}(\theta)=\frac{\exp \left(\tau_{i j r}\right)}{1+\exp \left(\tau_{i j r}\right)} \\
\tau_{i j r}=\overline{\tau_{i j}}+\sum_{m} a_{j m}^{\prime} \kappa_{i m r}
\end{gathered}
$$

(b) Given $\phi_{i j r}(\theta)$ and $u_{i j r}$ construct a $J$ dimensional Bernoulli vector, $b_{i r}(\theta)$. This defines the choice set $\mathcal{S}_{r}$ for the $r$ th loop, where the $j$ th element is determined according to

$$
b_{i j r}= \begin{cases}1 & \text { if } \phi_{i j r}(\theta)>u_{i j r} \\ 0 & \text { if } \phi_{i j r}(\theta) \leq u_{i j r}\end{cases}
$$

Define $b_{i r}^{0}$ to be the Bernoulli vector generated from the initial choice of parameters, $\theta_{0}$.

(c) Calculate

$$
P_{i j r}(\theta)=\frac{\exp \left\{\delta_{j}+\mu_{i j r}\right\}}{y_{i}^{\alpha}+\Sigma_{k: b_{i r, k}^{0}=1} \exp \left\{\delta_{k}+\mu_{i k r}\right\}}
$$

where $\mu_{i j r}$ is value of $\alpha \ln \left(y_{i}-p_{j}\right)+\sum_{k} x_{j k}\left(\sigma_{k} \nu_{i k r}+\sum_{d} \Omega_{k d} D_{i d}^{s}\right)$ given the $r$ th draw and $\theta$.

(d) Calculate

$$
s_{i j r}(\theta)=\Pi_{l \in \mathcal{S}_{r}} \phi_{i l} \Pi_{k \notin \mathcal{S}_{r}}\left(1-\phi_{i k}\right) \frac{P_{i j r}(\theta)}{\phi_{i r}^{0}\left(\theta_{0}\right)}
$$

where $\phi_{i r}^{0}\left(\theta_{0}\right)$ is the value of $\Pi_{l \in \mathcal{S}_{r}} \phi_{i l} \Pi_{k \notin \mathcal{S}_{r}}\left(1-\phi_{i k}\right)$ using the initial choice set evaluated at the initial value of the parameters, $b_{i r}^{0}$.

7. Calculate the simulator for the choice probability

$$
\widehat{s_{i j}}=\frac{1}{R} \sum_{r} s_{i j r}
$$

The firm choice probability (used in the micro moments) is

$$
\widehat{B}_{i f}=\sum_{j \in \mathcal{J}_{f}} \widehat{s_{i j}}
$$




\section{Appendix D. Preliminary Regressions}

\begin{tabular}{|c|c|c|c|c|c|c|c|}
\hline \multirow[b]{2}{*}{ Explanatory Variable } & \multicolumn{7}{|c|}{ Dependent Variable: Purchased PC in Last 12 Months } \\
\hline & Coefficient & Std. Error & Coefficient & Std. Error & Coefficient & & Std. Error \\
\hline Constant & -1.5549 ** & $(0.1399)$ & $-1.5133^{* *}$ & $(0.1376)$ & -1.4907 & ** & $(0.1383)$ \\
\hline age & $0.0141 * *$ & $(0.0058)$ & 0.0140 ** & $(0.0058)$ & 0.0132 & ** & $(.0058)$ \\
\hline age squared & $-0.0002 * *$ & $(0.0001)$ & $-0.0002 * *$ & $(0.0001)$ & -0.0002 & ** & $(.00006$ \\
\hline edusp (education if $<11$ ) & -0.0585 ** & $(0.0075)$ & $-0.0588^{* *}$ & $(0.0075)$ & -0.0609 & ** & $(.0074)$ \\
\hline eduhs (=1 if highest edu 12 years) & $-0.3427^{* *}$ & $(0.0503)$ & $-0.3441^{* *}$ & $(0.0502)$ & -0.3579 & ** & $(.0500)$ \\
\hline eduad ( $=1$ if highest edu $1-3$ college) & $-0.1735^{* *}$ & $(0.0466)$ & $-0.1715^{* *}$ & $(0.0465)$ & -0.1838 & ** & $(.0463)$ \\
\hline edubs ( $=1$ if highest edu college grad) & -0.1028 ** & $(0.0398)$ & -0.1008 ** & $(0.0398)$ & -0.1023 & ** & $(.0396)$ \\
\hline married $(=1$ if married $)$ & 0.1082 ** & $(0.0307)$ & $0.1067^{* *}$ & $(0.0306)$ & 0.1036 & ** & $(.0304)$ \\
\hline hh size (household size) & 0.0660 ** & $(0.0093)$ & 0.0660 ** & $(0.0093)$ & 0.063 & ** & $(.0092)$ \\
\hline inclow $(=1$ if income $<\$ 60,000)$ & -0.1436 ** & $(0.0305)$ & -0.1438 ** & $(0.0303)$ & -0.1586 & ** & $(.0301)$ \\
\hline inchigh ( $=1$ if income $>\$ 100,000$ ) & $0.1067^{* *}$ & $(0.0406)$ & $0.1093^{* *}$ & $(0.0405)$ & 0.1042 & ** & $(.0403)$ \\
\hline malewh ( $=1$ if male and white) & $0.0834^{* *}$ & $(0.0283)$ & 0.0828 ** & $(0.0283)$ & 0.0927 & ** & $(.0282)$ \\
\hline $\operatorname{mag} 1(=1$ if magazine quintile $=1)$ & -0.0383 & $(0.0325)$ & -0.0338 & $(0.0321)$ & & & \\
\hline mag 2 (=1 if magazine quintile=2) & 0.0482 & $(0.0306)$ & 0.0497 * & $(0.0304)$ & & & \\
\hline $\mathrm{np} 1(=1$ if newspaper quintile $=1)$ & 0.0176 & $(0.0308)$ & & & & & \\
\hline np 2 (=1 if newspaper quintile $=2$ ) & -0.0059 & $(0.0334)$ & & & & & \\
\hline tv 1 (=1 if television quintile=1) & -0.1264 ** & $(0.0627)$ & $-0.1240 * *$ & $(0.0626)$ & & & \\
\hline tv 2 (=1 if television quintile=2) & -0.0664 ** & $(0.0314)$ & $-0.0657^{* *}$ & $(0.0314)$ & & & \\
\hline radio 1 (=1 if radio quintile=1) & 0.0856 & $(0.0549)$ & & & & & \\
\hline radio 2 ( $=1$ if radio quintile $=2$ ) & 0.0116 & $(0.0264)$ & & & & & \\
\hline Log Likelihood & -6479 & & -6481 & & -6536 & & \\
\hline Likelihood Ratio Test Statistic & & & -4.7 & & -114.6 & & \\
\hline Prob>Test Statistic & & & 0.4538 & & 0.0000 & & \\
\hline
\end{tabular}

Note: These results use the complete Simmons data set; sample size 20,100. The first specification is the unrestricted model to which I compare the

other specifications. ${ }^{*}$ indicates significant at the $5 \%$ level; * significant at the $10 \%$ level.

Table D1: Probit Estimates of Purchase Probabilities

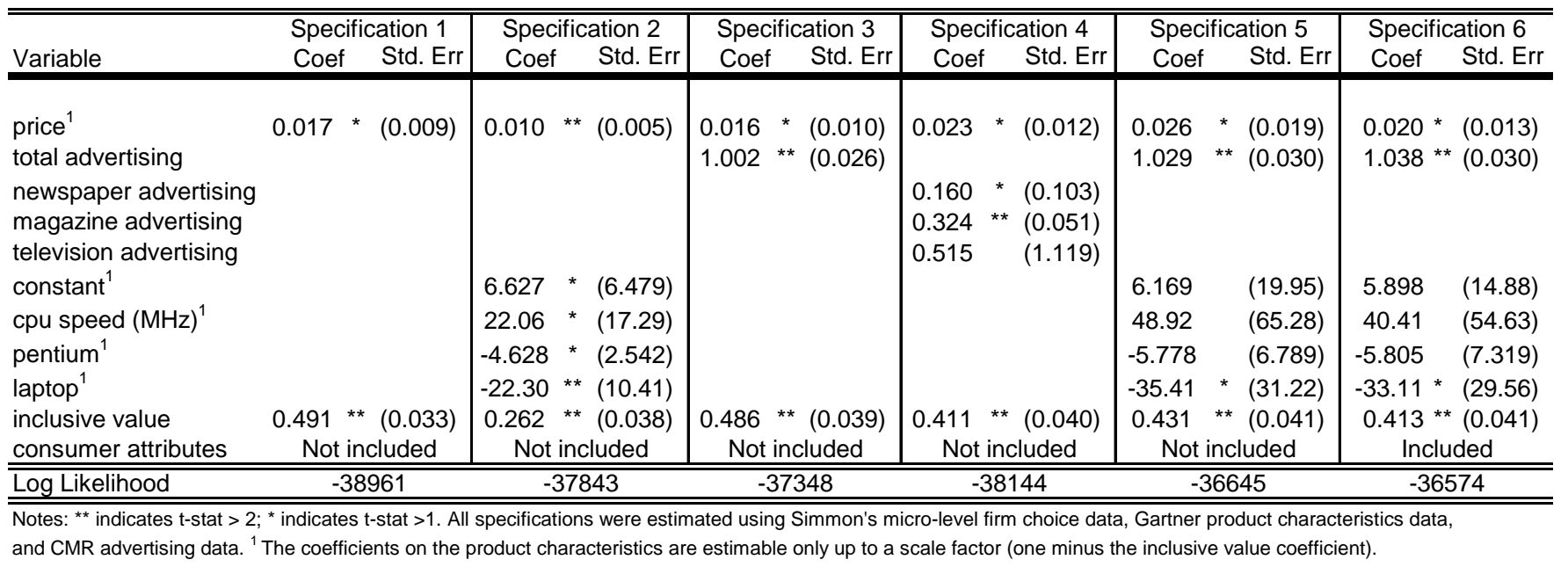

Table D2: Preliminary Nested Logit Estimates 


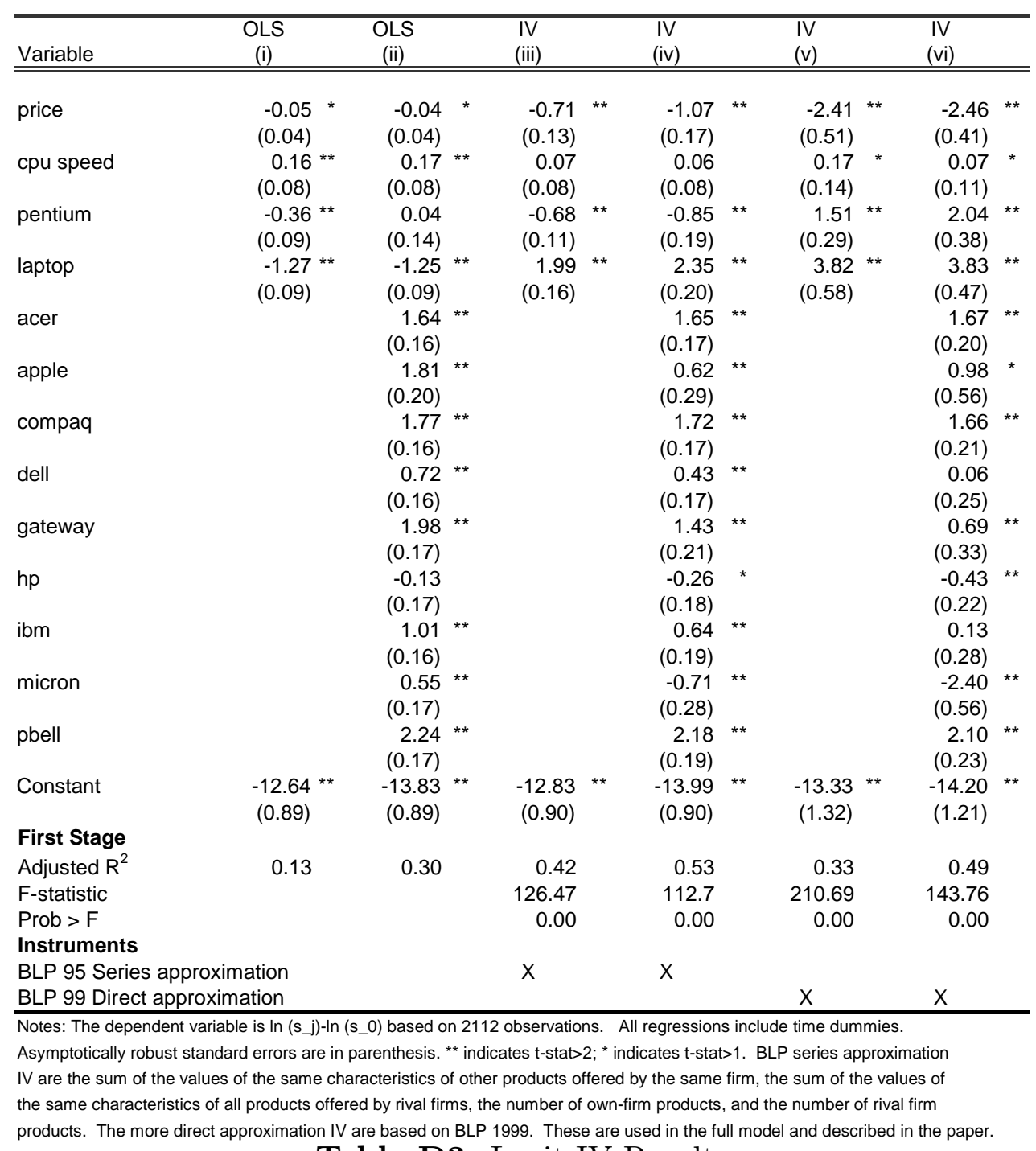

Table D3: Logit IV Results 


\section{References}

Ackerberg, D. (2001): Empirically Distinguishing Informative and Prestige Effects of Advertising, RAND Journal of Economics, 32, 100-118.

Ackerberg, D. (2003): Advertising, Learning, and Consumer Choice in Experience Goods Markets: A Structural Empirical Examination, International Economic Review, 44, 1007-1040.

Anand, B., and R. Shachar (2004): “Advertising, the Matchmaker," Harvard Business School Working Paper No. 02-057.

Anderson, S., and A. de Palma, and J.F. Thisse (1989): Demand for Differentiated Products, Discrete Choice Models, and the Characteristics Approach, Review of Economic Studies 56, 21-35.

Andrews, D. (1988): Chi-square Diagnostic Tests for Econometric Models, Journal of Econometrics, 37, 135-156.

Bajari, P., and C. L. Benkard (2005): Demand Estimation with Heterogenous Consumers and Unobserved Product Characteristics: A Hedonic Approach, Journal of Political Economy, 113, 1239-1276.

Berry, S. (1994): Estimating Discrete Choice Models of Product Differentiation, Rand Journal of Economics, 25, 242-262.

Berry, S., J. Levinsohn, and A. Pakes (1995): Automobile Prices in Market Equilibrium, Econometrica, 63, 841-890.

Berry, S., J. Levinsohn, and A. Pakes (1999): Voluntary Export Restraints on Automobiles: Evaluating a Trade Policy, American Economic Review, 89, 400-430.

Berry, S., J. Levinsohn, and A. Pakes (2004): Differentiated Products Demand Systems from a Combination of Micro and Macro Data: The New Car Market, Journal of Political Economy, 112, 68-105.

Bresnahan, T. (1989): Empirical Studies of Industries with Market Power, in Handbook of Industrial Organization, ed. by R. Schmalensee and R. Willig. North-Holland, New York and Oxford: Elsevier Science Publishers, Chapter 17.

Chamberlain, G. (1987): Asymptotic Efficiency in Estimation with Conditional Moment Restrictions, Journal of Econometrics, 34, 305-344.

Chiang, J., S. Chib, and C. Narasimhan (1999): Markov Chain Monte Carlo and Models of Consideration Set and Parameter Heterogeneity, Journal of Econometrics, 89, 223248.

Ching, A., T. Erdem, and M. Keane (2007): The Price Consideration Model of Brand Choice," forthcoming, Journal of Applied Econometrics

Erdem, T., and M. Keane (1996): Decision-making Under Uncertainty: Capturing Dynamic Brand Choice Processes in Turbulent Consumer Goods Markets, Marketing Science, $15,1-20$.

Geweke, J. (1988): Antithetic Acceleration of Monte Carlo Integration in Bayesian Inference, Journal of Econometrics, 38, 73-89.

Goeree, M.S. (2002): "Informative Advertising and the US Personal Computer Market: A Structural Empirical Examination," Ph.D. Dissertation, University of Virginia.

Goeree, M.S. (2003): "Was Mr. Hewlett Right? Mergers, Advertising, and the PC Industry," Unpublished Manuscript, Claremont McKenna College. 
Goeree, M.S. (2008): "Limited Information and Advertising in the US Personal Computer Industry, Supplementary Material: Miscellaneous," Econometrica Supplementary Material, http://www.econometricsociety.org/ecta/supmat/

Gourieroux, C., A. Monfort, E. Renault, and A. Trognon (1987): Generalized Residuals, Journal of Econometrics, 34, 5-32.

Grossman, G., and C. Shapiro (1984): Informative Advertising with Differentiated Products, Review of Economic Studies, 51, 63-82.

Hendel, I. (1999): Estimating Multiple-Discrete Choice Models: An Application to Computerization Returns, Review of Economic Studies, 66, 423-46.

Leslie, P. (2004): Price Discrimination in Broadway Theater, Rand Journal of Economics, $35,520-541$.

Mehta, N., S.Rajiv, and K. Srinivasan (2003): Price Uncertainty and Consumer Search: A Structural Model of Consideration Set Formation, Marketing Science, 22, 58-84.

Milgrom, P., and J. Roberts (1986): Price and Advertising Signals of Product Quality, Journal of Political Economy, 94, 796-821.

Nevo, A. (2000): A Practitioner's Guide to Estimation of Random Coefficients Logit Models of Demand, Journal of Economics and Management Strategy, 9, 513-548.

Nierop, E., R. Paap, B.Bronnenberg, P. Franses, and M. Wedel (2005): "Retrieving Unobserved Consideration Sets from Household Panel Data," UCLA Anderson Working Paper.

Pakes, A., and D. Pollard (1989): Simulation and the Asymptotics of Optimization Estimators, Econometrica, 57, 1027-1057.

Petrin, A. (2002): Quantifying the Benefits of New Products: The Case of the Minivan, Journal of Political Economy, 110, 705-729.

Rivers, D., and Q. Vuong (1988): Limited Information Estimators and Exogeneity Tests for Simultaneous Probit Models, Journal of Econometrics, 39, 347-366.

Shum, M. (2004): Does Advertising Overcome Brand Loyalty? Evidence from the Breakfast Cereals Market, Journal of Economics and Management Strategy, 13, 241-272.

Smith, R.J., and R. Blundell (1986): An Exogeneity Test for a Simultaneous Equation Tobit Model with an Application to Labor Supply, Econometrica, 54, 679-85.

Stern, S. (1997): Simulation-Based Estimation, Journal of Economic Literature, 35, 20062039.

Stern, S. (2000): Simulation Based Inference in Econometrics: Motivation and Methods, in Simulation-Based Inference in Econometrics: Methods and Applications, ed. by Mariano, Weeks, and Schuermann. Cambridge, U.K.: Cambridge University Press.

Vuong, Q.H. (1989): Likelihood Ratio Tests for Model Selection and Non-Nested Hypotheses, Econometrica, 57, 307-333.

Wooldridge, J. (2002): Econometric Analysis of Cross Section and Panel Data, Cambridge M.A.: MIT Press. 